\title{
Regard sur les formes dans la musique rituelle du bouddhisme tibétain
}

\section{Mireille Helffer}

\section{Q OpenEdition}

1 Journals

Édition électronique

URL : http://journals.openedition.org/ethnomusicologie/566

ISSN : 2235-7688

\section{Éditeur}

ADEM - Ateliers d'ethnomusicologie

Édition imprimée

Date de publication : 1 janvier 2004

Pagination : 261-295

ISBN : 2-8257-0910-7

ISSN : $1662-372 X$

\section{Référence électronique}

Mireille Helffer, "Regard sur les formes dans la musique rituelle du bouddhisme tibétain », Cahiers

d'ethnomusicologie [En ligne], 17 | 2004, mis en ligne le 13 janvier 2012, consulté le 21 décembre 2020. URL : http://journals.openedition.org/ethnomusicologie/566

Ce document a été généré automatiquement le 21 décembre 2020.

Tous droits réservés 


\title{
Regard sur les formes dans la musique rituelle du bouddhisme tibétain
}

\author{
Mireille Helffer
}

La cérémonie commença à onze heures de la nuit; les lamas vinrent se ranger en rond au fond de la tente, armés de cymbales, de conques marines, de cloches, de tambourins et de divers instruments de leur bruyante musique [...]. Au signal donné, l'orchestre exécuta une ouverture musicale capable d'effrayer le diable le plus intrépide. Les hommes noirs et séculiers battaient des mains en cadence, pour accompagner le son charivarique et les hurlements des prières. (Huc 1987, Tome 1:

139-140).

1 Cette description peu enthousiaste du Père Huc dont les observations remontent au milieu du XIX ${ }^{\mathrm{e}}$ siècle n'a plus cours aujourd'hui. Depuis la deuxième moitié du $\mathrm{XX}^{\mathrm{e}}$ siècle, les connaissances relatives à la musique rituelle du bouddhisme tibétain se sont multipliées, d'une part grâce à la publication de nombreux documents sonores (en commençant par ceux que recueillit en 1961 P. Crossley-Holland, le pionnier des études relatives à la musique tibétaine), d'autre part en raison du rapide développement des monastères tibétains en exil.

2 Cette situation nouvelle a incité quelques chercheurs musicologues, fascinés par ces musiques venues $\mathrm{du}$ Toit $\mathrm{du}$ monde, à développer leur connaissance du bouddhisme tibétain et à apprendre la langue tibétaine dont l'étude devenait progressivement possible dans le cadre des institutions universitaires.

3 Comme j'ai déjà eu l'occasion de l'expliquer dans plusieurs publications (notamment Helffer 1991 et 1995), depuis 1989, j'ai pour ma part choisi de me consacrer aux musiques pratiquées au sein d'un monastère de la tradition nyingmapa, le monastère de Shechen ( = 
Zhe-chen), fondé en 1735 dans la région du Kham (province chinoise du Sichuan), et en exil au Népal à Bodnath depuis 1985.

La fréquentation régulière $\mathrm{du}$ Shechen népalais depuis plus de quinze ans et l'accueil chaleureux des autorités monastiques, auxquels s'ajoutait l'aide éclairée du moine français Matthieu Ricard, m'ont permis d'assister à de nombreux rituels, de réaliser quantité d'enregistrements et d'étudier les textes relatifs aux répertoires musicaux du monastère. La réunion de tous ces facteurs favorables m'incite aujourd'hui à initier une réflexion sur l'organisation formelle des différents répertoires musicaux auxquels il est fait appel dans la pratique liturgique de ce monastère. Je m'appuierai principalement sur les données recueillies durant la célébration du grand rituel qui marque la fin de l'année tibétaine, le gtor-zlog ou dgu-gtor, qui se déroule du $23^{\mathrm{e}}$ au $29^{\mathrm{e}}$ jour $\mathrm{du} 12^{\mathrm{e}}$ mois $\mathrm{du}$ calendrier tibétain, autrement dit en février-mars du calendrier occidental (Helffer 2004).

\section{Les sources tibétaines écrites utilisées pour la célébration du gtor-zlog}

5 Avant de rentrer dans le vif du sujet, il me semble indispensable de présenter brièvement le matériel sur lequel est fondée ma réflexion.

Comme tout rituel du bouddhisme tibétain, le gtor-zlog est basé sur un assemblage de textes composés par des maîtres de la tradition. Il a pour but de prévenir les difficultés qui pourraient surgir dans l'année à venir en s'adressant à la divinité d'élection (yi-dam) du monastère - une forme particulière de Ghin-rje-gshed (skt. Yamantaka) - et à son acolyte Las-gshin, et en s'assurant la bonne volonté et l'appui des neuf protecteurs (chosskyong) de l'ordre nyingmapa et de leurs assistants (rjes-'brang).

7 Les textes réunis pour ce faire ont pour auteurs deux maîtres éminents de l'école nyingmapa: Gter-bdag gling-pa (1646-1714), fondateur du monastère de Smin-grol-gling au Tibet central, et le frère de celui-ci, Lochen Dharmashri (1654-1717/18).

Quant à l'aspect sonore que doit revêtir le rituel, il est consigné dans différents manuels manuscrits de notations musicales (dbyangs-yig), élaborés par les maîtres de chant (dbumdzad) et regroupés dans une publication récente du monastère (S.T.1 de la bibliographie). Il s'agit en premier lieu du manuel concernant le culte rendu aux neuf protecteurs et à leurs assistants, intitulé « Ce qui fait la joie des protecteurs de la doctrine ( "Chos-skyong rnams kyi dbyangs-yig «bstan-srung dgyes-par byed-pa»,S.T.1: 267-425), dont le colophon précise qu'il fut composé en 1822, et en second lieu du manuel consacré au culte de la «divinité d'élection» (yi-dam) du monastère - Gshin-rje-gshed, sous sa forme de dregs-pa 'joms byed -, intitulé "le rugissement du lion» (Gshin-rje dregs-joms byed kyi dbyangs-yig "seng-ga'i nga-ro», S.T.1: 509-630). L'accès à ces manuels était jadis réservé aux maittres de chant; mais, depuis la publication qui en a été faite en Inde, leur consultation s'est trouvée facilitée et de nombreux jeunes moines s'y réfèrent. 
Fig. 1 : Les gâteaux rituels (gtor-ma) représentant Gshin-rje-gshed et Las-gshin durant la célébration du gtor-zlog

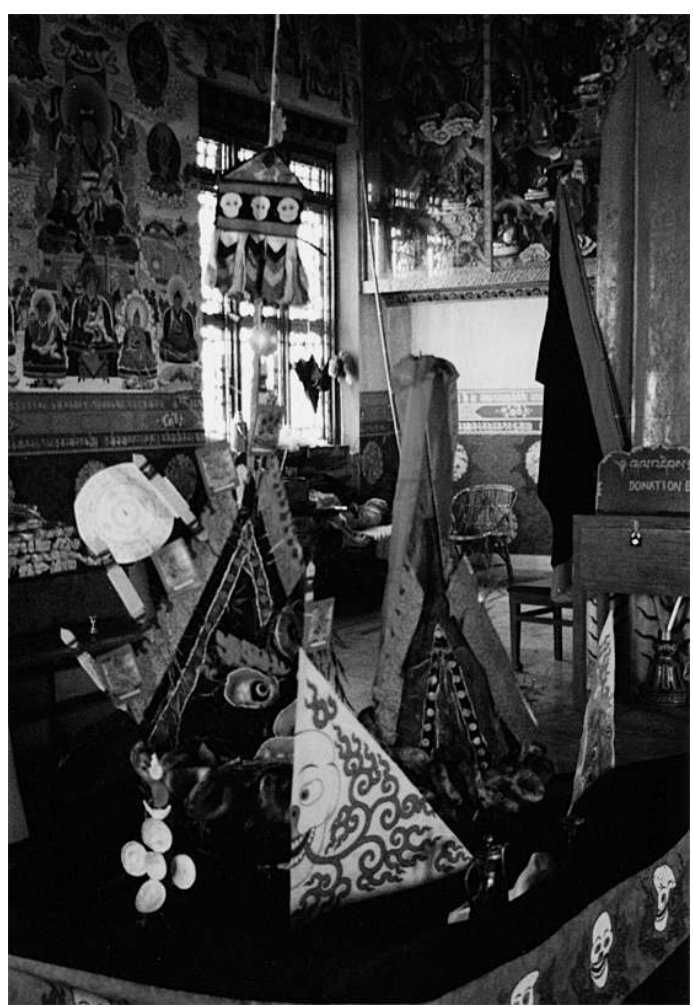

Fig. 2: le maître de chant 'Gyur-med 'jigs-med s'accompagnant aux cymbales sbug-chal

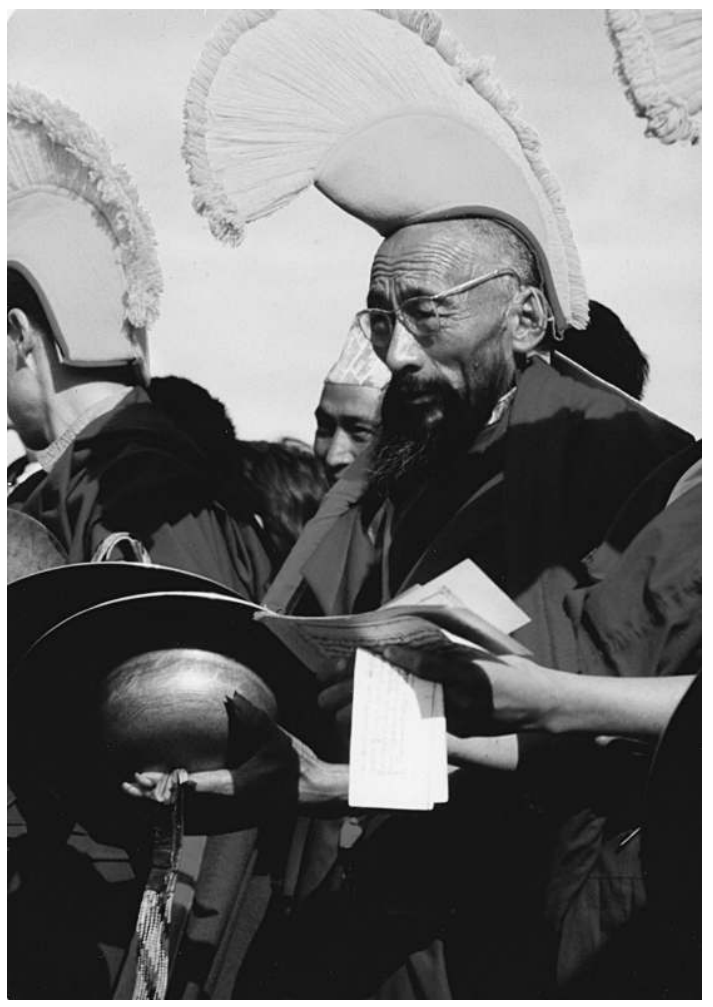


Ces précieux documents sont complétés par des «modes d'emploi» (lag-len ou phyag-len) explicitant l'usage liturgique des textes précédents; ils ont récemment été publiés en deux volumes par les soins du monastère et regroupent cinq recueils de ce type, parmi lesquels celui qui concerne précisément le rituel du gtor-zlog ('Jam-dpal Gshin-rje dregs-pa joms-byed kyi gtor-zlog lag-len, S.T.2, vol. 2: 61-190).

Il faut en outre signaler l'existence de quelques feuillets manuscrits concernant les notations pour le jeu des trompes dung-chen et rkang-gling (S.T.6)

11 A ces documents écrits se sont ajoutés, au cours des années (notamment en 1989, 1991, 1993, 1995 et 1997), les documents sonores que j'ai pu recueillir aussi bien pendant le rituel, que hors situation, à la demande, répondant ainsi au vœu exprimé par l'abbé Rabjam Rinpoche, soucieux de conserver la tradition du monastère de Shechen et d'en constituer les archives sonores. De cette préoccupation a témoigné l'appel fait à d'anciens maîtres de chant (dbu-mdzad, prononcé umze) du Shechen tibétain pour qu'ils viennent transmettre aux jeunes générations la tradition orale dont ils étaient les dépositaires: on a ainsi vu se succéder de 1990 à 1993 le dbu-mdzad 'Gyur-med 'jigs-med, puis de 1993 à 1995 le dbu-mdzad Nor-bzang; c'est auprès d'eux que les jeunes moines qui exercent aujourd'hui la fonction de dbu-mdzad ont été formés ${ }^{1}$.

12 L'existence de ces différentes sources, exprimant par écrit le point de vue des experts tibétains et, au travers des enregistrements, la traduction sonore qui en est donnée, offre une possibilité exceptionnelle d'élucider quelques unes des formes dans lesquelles se développe la musique rituelle du bouddhisme tibétain, tant au niveau de la production vocale qu'à celui de l'important dispositif instrumental mis en œuvre.

\section{L'énonciation des textes et les composantes de la musique vocale}

Puisque c'est autour des textes que s'organise le rituel, il est nécessaire de rappeler les éléments formels qui les composent. Le lecteur et surtout les acteurs du rituel doivent en effet naviguer entre des feuillets multiples imprimés (en écriture dbu-chen) ou manuscrits (en écriture dbu-med).

\section{La structure des textes}

Des syllabes-germes (skt bija / tib. sa-bon) monosyllabiques héritées du sanscrit, telles OM, HUM, BHYO, HRIH, BAI..., marquent le début de chaque texte. Elles constituent la forme sonore de la divinité et servent de point de départ au maître de chant.

En outre, de nombreux mantras formés de plusieurs syllabes sont accumulés en divers points du rituel:

- Les textes en prose

- Les textes en vers ( $r k a n g-p a)$, qui relèvent du domaine poétique, (snyan-ngag). Vu la place prépondérante qu'ils occupent dans les rituels, il est nécessaire de rappeler quelques-uns des principes qui les régissent, d'autant plus que la poétique tibétaine ne connaît pas la rime et repose essentiellement sur le rythme, l'accentuation et la césure.

16 Etant donné que les mots de la langue tibétaine sont généralement monosyllabiques (chaque syllabe étant séparée de sa voisine par un point ${ }^{\circ}$ placé en haut et à droite ou par 
un trait), mais sont aussi disyllabiques, par commodité, j'ai retenu ici le terme de pied pour désigner tout groupement de deux syllabes; cela étant admis, je considère que la segmentation $\mathrm{du}$ vers s'opère en pieds disyllabiques avec accentuation des syllabes impaires, ce qui peut faire penser à un rythme trochaïque, bien que, contrairement à la poésie sanscrite qui lui servait de modèle, le tibétain ne connaisse pas l'opposition longue/brève.

Dans la littérature religieuse et en particulier dans les textes rituels qui nous occupent, on observe une prédominance absolue de vers à 7 et 9 syllabes qui peuvent être regroupés en strophes de deux ou quatre vers.

Ceci donne pour un vers à 7 syllabes: trois pieds disyllabiques + un pied catalectique, ou deux pieds disyllabiques + un trisyllabe, avec dans tous les cas une césure $4 / 3$ :

$\mathrm{xx} / \mathrm{xx} / / \mathrm{xx} / \mathrm{x}$

$\mathrm{x} \mathrm{x} / \mathrm{x} \mathrm{x} / / \mathrm{x} \mathrm{x} \mathrm{x}$

19 Un exemple, parmi quantité d'autres, en est fourni par le texte d'une invitation pressante au «démon-planète» (Gza'-bdud/skt Rahula), choisi en raison du rôle métrique des trisyllabes-onomatopées qui occupent la deuxième partie de plusieurs vers:

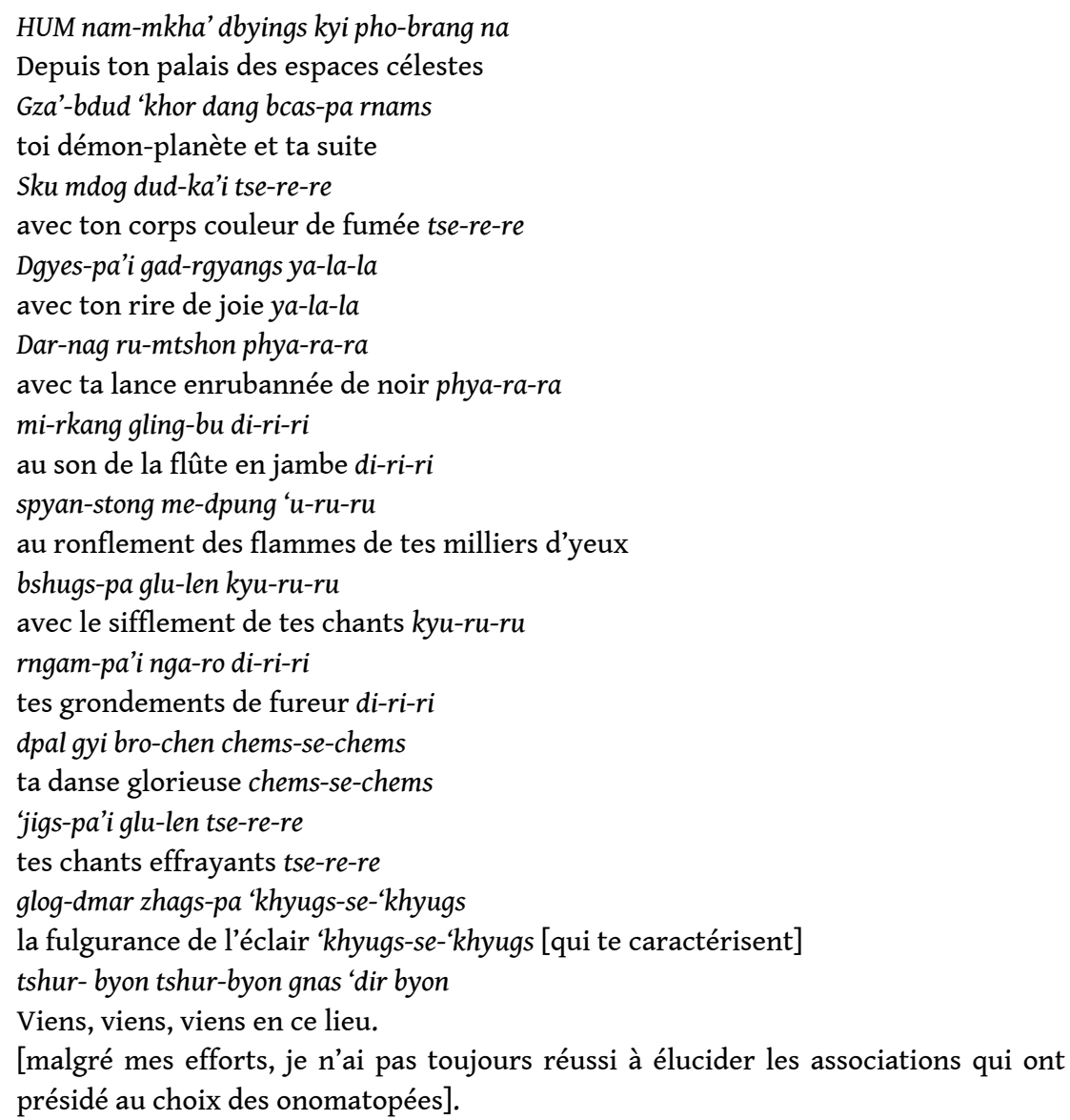

Pour les vers à 9 syllabes, on constate la présence de quatre pieds disyllabiques et un pied catalectique avec césure après la sixième syllabe, ou de trois pieds disyllabiques suivis de la figure de style que constitue le trisyllabe-onomatopée: $\mathrm{x} x / \mathrm{x} x / \mathrm{xx} / / \mathrm{xxx}$ 
21 L'énumération des offrandes ( $m c h o d-p a)$ destinées à une forme particulière du protecteur Mgon-po témoigne de jeux sonores identiques à l'exemple précédent; dans ce cas, la césure intervient après les six premières syllabes du vers (R. Kohn, 1989: 629-630).

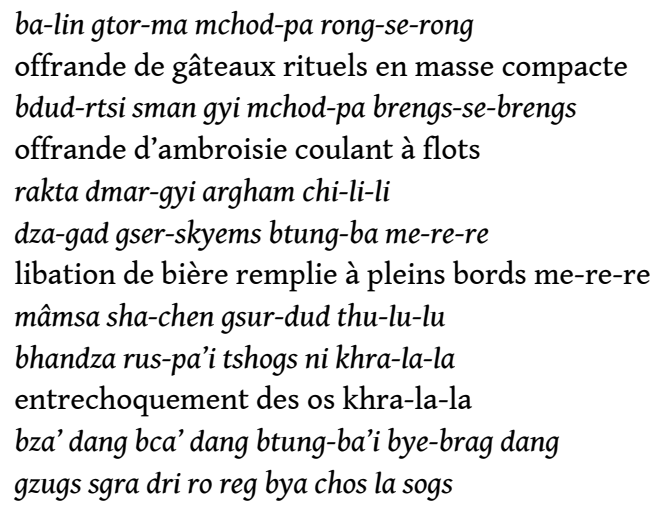

22 Le recours à de nombreux trisyllabes-onomatopées que les grammairiens d'aujourd'hui classent dans les « impressifs» et dont la traduction demeure difficile n'est pas propre aux textes utilisés à Shechen, mais se retrouve abondamment dans la littérature religieuse ou profane, comme le confirme un passage de rituel gelugpa en l'honneur du protecteur Mgon-po:

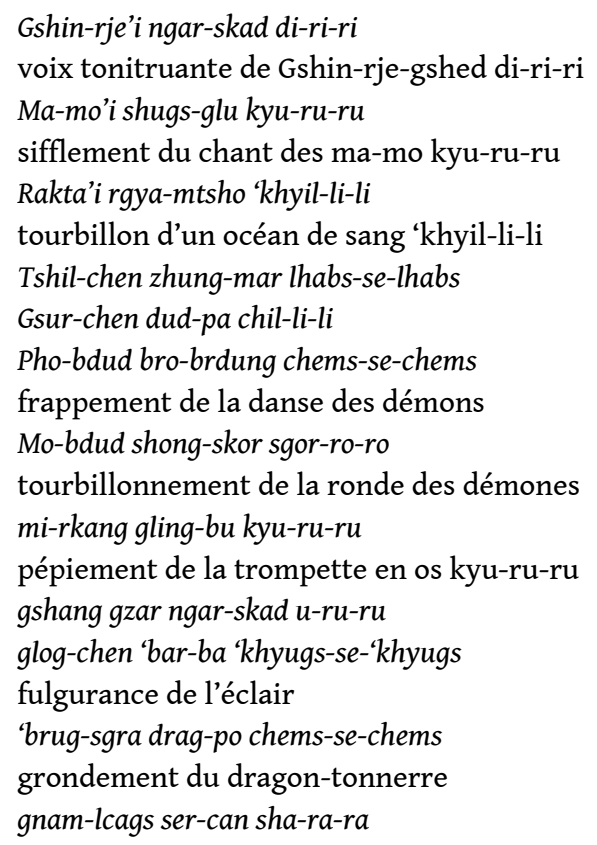

\section{Les modalités de l'énoncé des textes}

La connaissance des textes (en théorie appris par cœur) ne suffit pas, il faut aussi savoir comment les énoncer, exercice auquel les jeunes moines sont entraînés dès l'enfance en participant (sans y comprendre grand-chose) aux interminables rituels qui occupent le calendrier liturgique.

Une place à part doit être faite à l'énonciation des nombreux mantras (sngags) qui sont récités pendant le rituel et surtout au mantra-racine (rtsa-sngags) dont la récitation ne doit pas être interrompue et se poursuit jour et nuit pendant les huit jours du rituel. Il s'agit en l'occurrence du mantra de Gshin-rje-gshed (skt. Yamantaka), la forme 
« courroucée» (drag-po) du bodhisattva Manjushri, répété à tue-tête et fortement scandé par un groupe de moinillons, et que la notation segmente ainsi (S.T.1: 537):

OM A-KRO-TE-/ KA / YA-MAN-TA-KA KÂ LA RU PA HRIH SHRI

suivi de: A PA YE / MA MA KRO-DHI-SHVA-RI SA-MA-YA HUM PHAT//

Le sgrub-pa'i rtsa-sngags, c'est-à-dire le mantra-racine de ce rituel de grande évocation ( sgrub-chen) est plus bref mais joint les noms sanskrits des formes paisibles et courroucées du bodhisattva; il se limite à OM MANJUSHRI YAMANTAKA HUM PHAT //

Pour tous les autres textes, la terminologie tibétaine distingue plusieurs modalités d'énonciation qui ont été magistralement décrites dans une contribution déjà ancienne de Ter Ellingson (1979b) et qui se retrouvent dans les manuels de Shechen, où sont distingués:

- la simple récitation ('don-pa) effectuée recto tono selon plusieurs modalités;

-lhug-'don pour la prose;

•'dur-'don pour une récitation " galopante», qui peut être très rapide;

-drag-'don pour une récitation fortement scandée.

- un mode de psalmodie à caractère syllabique, effectué sur une mélodie-type simple transmise oralement et connue de tous, correspondant le plus souvent à une strophe de deux vers, reprise sans modification jusqu'à la fin du texte.

- un mode de psalmodie plus élaboré qui peut faire l'objet de notation, désigné à Shechen par le terme gdangs et dans lequel il n'y a pas introduction de syllabes étrangères au texte, mais qui est ponctué par le jeu des cymbales/tambours. Tel est le cas pour un sman-gdangs noté S.T.1: 529 avec frappe une syllabe sur deux; pour le gdab-gdangs noté S.T.1: 566 avec une frappe sur chaque syllabe; tandis que le bar-gdangs mentionné S.T.1: 531 ne requiert aucune frappe.

- un mode de chant spécifique désigné par le terme dbyangs (dont le sens premier est «voyelle») qui constitue la solennisation maximum des textes. La composition de ces dbyangs, souvent désignés par des titres plus ou moins poétiques ou pompeux, est parfois attribuée à des maitres religieux qui en auraient eu, en quelque sorte, la vision sonore; les problèmes relatifs à leur transmission fidèle ont conduit les maîtres de chant à élaborer des systèmes de notations contenus dans les manuels de chant dbyangs-yig dont il y a lieu de préciser quelque peu le contenu.

Les manuels, propres à la tradition du monastère de Shechen, étaient, jusqu'à une date récente, conservés sous forme exclusivement manuscrite et, avant la publication qui en a récemment été faite en Inde (S.T.1), leur accès était réservé aux seuls maîtres de chant. Ils se présentent désormais sous la forme traditionnelle des ouvrages tibétains (dpe-cha) ${ }^{2}$; les folios écrits recto-verso en écriture dbu-med comportent cinq lignes et se lisent de gauche à droite et de haut en bas.

Les conventions adoptées par les rédacteurs des dbyangs-yig peuvent se résumer aux points suivants:

- Sur chaque ligne sont portées syllabes du texte et syllabes sans signification (tshig-lhad) dont le caractère phonétique dépend de la syllabe qui les précède: si les syllabes signifiantes sont en rouge, les tshig-lhad qui constituent le premier degré de la notation musicale des dbyangs sont portés en noir et inversement.

- Structure des tshig-lhad: écho de la syllabe qui précède / enchaînements conventionnels de groupes de voyelles tels que ha-ha-ha / ya-a / wa-i ya /

Le nombre de tshig-lhad peut être si grand que le sens du texte est complètement masqué à l'audition. 
- Des espacements interviennent pour marquer différents types de segmentation: césures (4/3 comme pour la métrique du vers, mais aussi $6 / 1$ ou d'autres combinaisons), unités de souffle et division en vers.

- Des petits cercles vides sont placés au-dessous des syllabes dont l'énoncé doit être souligné par une frappe des cymbales actionnées, rappelons-le, par le maître de chant. Ils peuvent être absents (pour les dbyangs les plus solennels), être placés sous chacune des syllabes, ou plus fréquemment sous une syllabe sur deux, et rarement sous la première et la dernière syllabe du vers [c'est notamment le cas pour le dbyangs d'hommage au protecteur Mgon-po à quatre visages (S.T.1: 283); le dbyangs de louange à Bar-ma nag-mo (S.T.1: 322); la louange à Khyab-'jug chen-po (S.T.1:334); l'invitation à Gshin-rje'i -gshed, pour ne retenir que quelques exemples (particulièrement abondants dans le manuel relatif à Gshin-rje-gshed avec: S.T.1: 543, 545-46, 551, 583-585 etc.)].

Soit l'invocation à Mgon-po à quatre visages, un des plus importants protecteurs du bouddhisme tibétain.

La notation qui en est proposée (S.T.1: 295) porte sur deux vers à 9 syllabes précédés de la syllabe-germe HUM: Dpal-chen mthu-stobs dbang-phyug 'khor bcas la / Ma-rung log 'dren dgra dang bgegs rnams kyi /

Elle est identique pour chacun des vers et truffée de syllabes sans signification tshig-lhad.

Fig. 3: Notation de Dpal-chen mthu-stobs (S.T.1: 295)

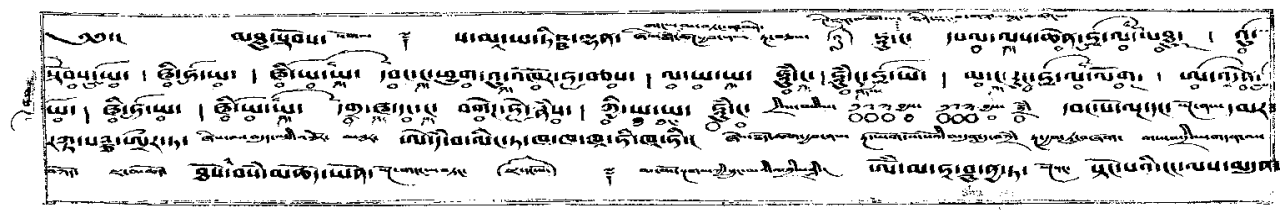

31 Dans la transcription qui va suivre, les syllabes du texte sont portées en italiques et les tshig-lhad en romains; les intervalles marquant la segmentation sont exprimés par un [/] et les cercles de frappes de cymbales par un simple point $\left[^{\circ}\right]$, ce qui donne:

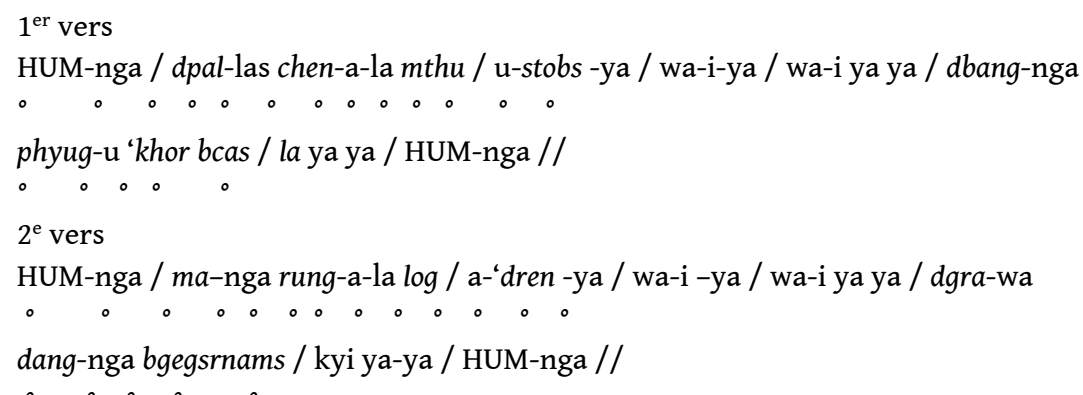

A ces conventions s'ajoutent des signes neumatiques parcimonieusement distribués, placés au-dessus ou en dessous des syllabes (qu'elles soient signifiantes ou non signifiantes), si celles-ci doivent faire l'objet d'une modification de la hauteur de référence ou de la couleur sonore à lui affecter.

Les enregistrements dont je dispose et notamment celui réalisé en 1995 par le dbu-mdzad Nor-bzang s'accompagnant lui-même aux cymbales, permettent d'affirmer que la présence d'une courbe placée au-dessus d'une syllabe indique une élévation de la hauteur du son par rapport à la note-référence $(+75 \mathrm{~Hz})$, tandis que des ondulations terminant 
l'un ou l'autre signe de notation indiquent un "tremblement», autrement dit un tremolo vocal plus ou moins accentué, que souligne le tracé des sonogrammes qui ont été réalisés.

Un deuxième exemple est fourni par l'invocation à Gshin-rje-gshed, exprimée elle aussi en vers à 9 syllabes et qui commence par les deux vers suivants:

Chos-dbyings mtha'-bral drag-po'i zhing-khams nas

Snying-rje rab 'khros 'Jam-dpal Gshin-rje-gshed...

Fig. 4: Notation de l'invocation à Gshin-rje-gshed (S.T.1: 519-521)

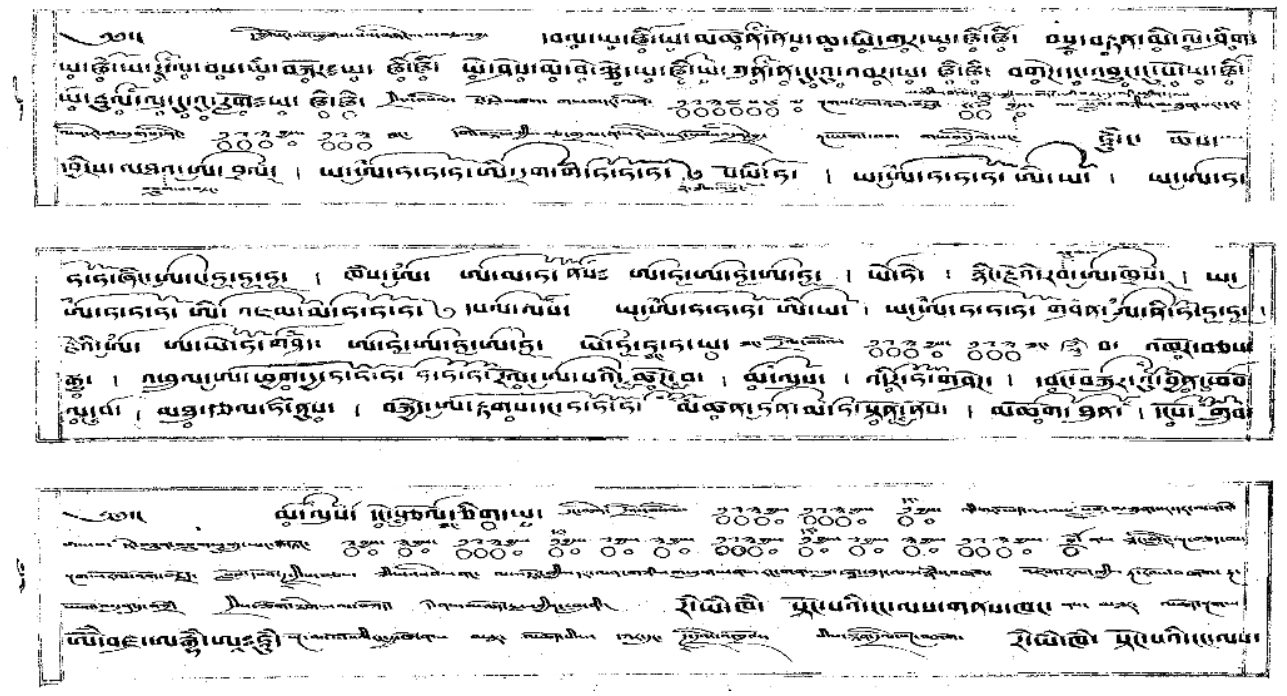

La notation qui en est proposée, identique pour chacun des vers, fait elle aussi une large place aux tshig-lhad, mais leur distribution est très différente (ils sont absents pour le début du vers et abondants à partir de la quatrième syllabe de chaque vers), ce qui induit une segmentation irrégulière du texte; seule la dernière syllabe de chaque vers est soulignée par une frappe de cymbales.

La transnotation proposée selon les principes adoptés ci-dessus donne:

$1^{\mathrm{er}}$ vers

HUM-nga / chos dbyings mtha'-a bral / ya-a ha-ha-ha / drag-ga ha-ha-ha po'i ha / ya-a

ha-

ha-ha-i-ya / zhing-a-nga ha-ha-ha / khams-a ama ha nas/ a-ha-ha a-ha /ye-he//

$2^{\mathrm{e}}$ vers

snying-rje'i rab-a khros / ya-a ha-ha-ha 'jam-ma ha-ha-ha dpal-las / ya-a ha-ha-ha-iya / ya-

a ha-ha-ha Gshin-a-ni ha-ha-ha / rje'i-a a-ye-ha gshed / a-ha a-ha a-ha / ye-ha ha-ha ya //

37 L'énonciation des quatre vers qui suivent se poursuit sur un ton de psalmodie simple, portant sur deux vers, incluant peu de tshig-lhad et prévoyant une frappe de cymbales une syllabe sur deux; la segmentation est irrégulière:

(1) 'khor bcas sgyu / 'phrul-a phyag-rgya ha-ha-ha ha-ha-ha rol-pa'i tshogs-ba /

(2) ma lus / 'dir gshegs / dbang bskur-u byin phob la-ba /

(3) mthu-rtsal nus / bskyed-a rtags dang ha-ha-ha mtshan-tan ma-a ston-nas / 
(4) mchog thun / dngos-sgrub ma lus / deng stsol cig-ya marquent clairement la ponctuation à effectuer par rapport aux textes en vers qui sont chantés, mais les manuels contenus dans S.T.1 comportent également de nombreuses indications relatives aux séquences rythmiques intercalées entre strophes ou parties de rituels, qui servent de signaux sonores ou qui sont organisées en pièces complexes portant éventuellement des titres.

Une fois encore, les enregistrements confirment la stabilité de la note de référence sur laquelle se base cette forme de "tone-contour melody» selon la terminologie d'Ellingson, tandis que les sonagrammes mettent en évidence la richesse des formants qui résultent des changements de voyelles dus à l'énoncé des tshig-lhad.

C'est en effet la place occupée par les tshig-lhad qui façonne en quelque sorte ces « chants à voyelles» ou chants « voyellisés» comme j'ai tendance à les appeler.

Seul l'examen systématique de toutes les notations proposées permettrait d'esquisser une théorie générale des formes illustrées dans les chants dbyangs; l'absence quasi absolue de chercheurs s'intéressant à ces questions l'a jusqu'à présent rendue impossible.

Même si les notations des dbyangs-yig concernent essentiellement la production vocale, ils fournissent en outre quantité de prescriptions relatives à l'instrumentation, aux interventions des différents instruments - avec conque (dung-dkar bcas), avec tambour damaru et clochette dril-bu (da-dril bcas), avec tambour (rnga bcas) -, aux formules rythmiques à exécuter, aux formules de trompettes rkang-gling à introduire, aux pièces pour hautbois rgya-gling ou trompes longues dung-chen préconisées à tel ou tel moment du rituel, etc. De plus, des commentaires en petits caractères sont parfois ajoutés entre les lignes pour rappeler certains aspects de la tradition orale (ou certaines modifications apportées par un des maîtres de chant).

L'examen de ces données, complété par l'étude des enregistrements qui en illustrent l'application, va permettre de dégager les caractéristiques formelles des diverses interventions instrumentales qui ponctuent et embellissent la proclamation des textes. Les remarques qui suivent porteront successivement sur la structuration du temps par le jeu des cymbales et tambours, les interventions des instruments dans lesquels on souffle, la coordination entre le jeu des différents instruments.

\section{La structuration du temps par le jeu simultané des cymbales et des tambours}

Comme il a déjà été signalé à propos du chant des dbyangs, les notations musicales Ces indications sont portées, comme on l'a vu à propos des chants, au moyen de petits cercles de différentes tailles, selon que la frappe doit être jouée $f$ ou $p$, mais ceux-ci sont alors surmontés d'un chiffrage indiquant la structuration de ces séquences et correspondant à la façon dont les tibétains « comptent».

\section{Les séquences rythmiques de base}

45 Parmi les séquences de base les plus fréquentes, on notera le «frapper trois» (gsumbrdung) et le «frapper neuf» (dgu-brdung), qui interviennent avec de nombreuses variantes. Pourtant le titre donné à ces séquences ne doit pas faire illusion. 

dans les diverses formulations qui peuvent être faites de gsum-brdung. Dans le cas le plus simple, gsum-brdung enchaîne trois coups $f$, suivis d'un coup $p$ (noté par la syllabe byas, prononcée djé), puis à nouveau trois coups $f$ :

123 byas 123

Cette formulation peut être prolongée par un coup faible:

123 byas 123 byas

Elle peut aussi être suivie d'un tremolo des cymbales exprimé par la syllabe thang:

123 byas 123 byas thang

Elle peut être précédée de deux syllabes hors-compte suivies d'un coup faible, ce qui donne un total de 12 frappes:

sbram sbram byas 123 byas 123 byas thang

\section{$<0$ oO 00 o O o O >}

123456789

A côté de ces formules de base, il existe également des formules-signaux pour marquer des transitions, mais elles ne font pas l'objet de notation graphique; c'est notamment le cas du zil-bebs qui se traduit par une succession de frappes de plus en plus rapprochées et de plus en plus piano et de bzhag-rol qui indique l'arrêt du jeu des cymbales qui doivent alors être déposées.

53 Le répertoire de Shechen comporte en outre plusieurs compositions développées qui enchaînent des séquences au sein desquelles la séquence à 9 frappes joue un rôle prédominant.

Un exemple en est fourni par la « bénédiction développée des cymbales» (rol-mo byin-rlabs rgyas-pa) qui figure dans un autre rituel et se présente comme suit:

Fig. 5: Notation de byin-rlabs rgyas-pa (S.T.1: 209)

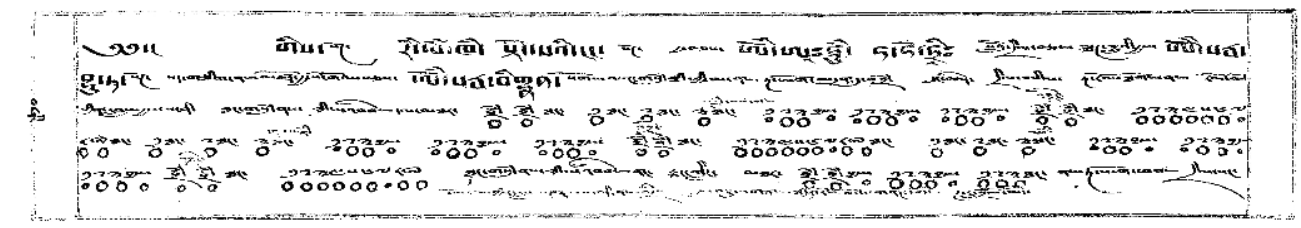


La transposition qui suit met en évidence la structure de la pièce, avec ses trois retours à la séquence à 9 frappes, séparés les uns des autres par des formulations de la séquence à 3 frappes diversement exprimées (avec ajout de tremolos thang, avec premier coup faible... ).

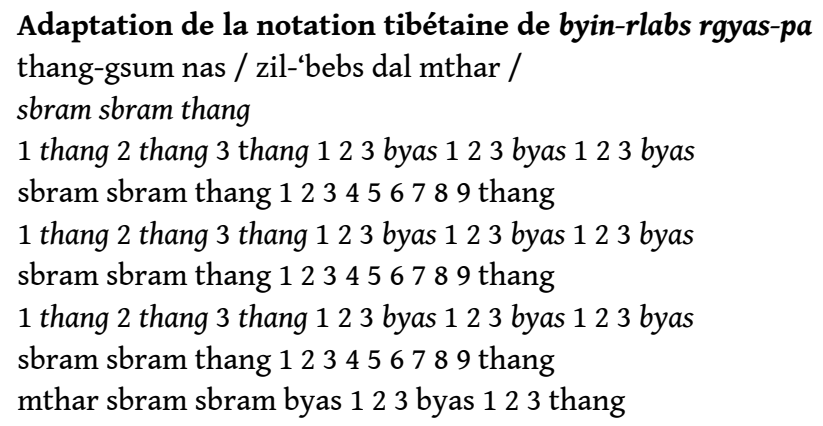

Même rôle préférentiel de la séquence à 9 frappes pour la « formulation développée de la musique de bon augure» (bkra-shis rol-mo rgyas-pa) exécutée à la fin de deux rituels différents et qui commence par trois énoncés successifs de dgu-brdung avant d'enchainer trois énoncés de 7 frappes, puis trois énoncés de 5 frappes et trois énoncés de 3 frappes, avant de terminer par une coda.

Fig. 6: Notation tibétaine de bkra-shis rol-mo rgyas-pa d'après S.T.1: 418

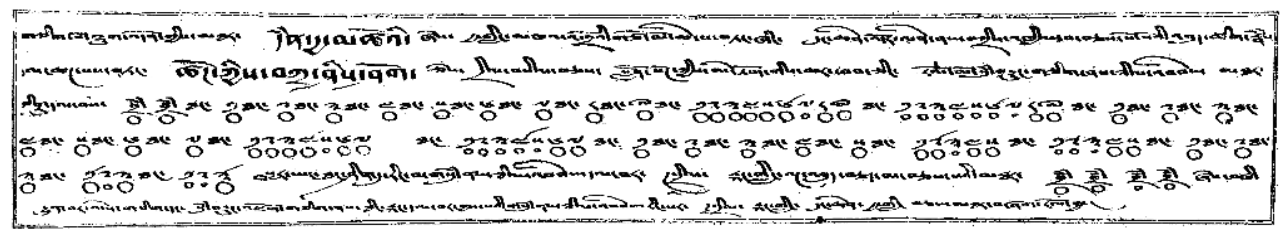

Adaptation de la notation tibétaine de bkra-shis rol-mo rgyas-pa

Sbram sbram thang

f 1 thang 2 thang 3 thang 4 thang 5 thang 6 thang 7 thang 8 thang 9 thang

mf 123456789 thang

p 123456789 thang

f 1 thang 2 thang 3 thang 4 thang 5 thang 6 thang 7 thang

mf 1234567

p 1234567

f 1 thang 2 thang 3 thang 4 thang 5 thang

mf 12345

p 12345

f 1 thang 2 thang 3 thang

$\mathrm{mf} 123$

p 123

[Coda] après trois fois thang, zil-'bebs suivi de bzhag-rol

N.B. un chiffre de petite taille indique un coup faible

Plus complexe encore s'avère la pièce intitulée « les neuf frappes du rgyal-po» - rgyal-po'i dgu-brdung - dédiée à Tsi'u dmar-po, qui appartient à la catégorie des divinités rgyal-po; elle prend place dans le rituel des protecteurs (chos-skyong) et le CD Sounds of Tibet: plage 4 en fournit un enregistrement recueilli en 1997. 
Fig. 7: Notation tibétaine de rgyal-po'i dgu-brdung selon S.T.1: 361-362

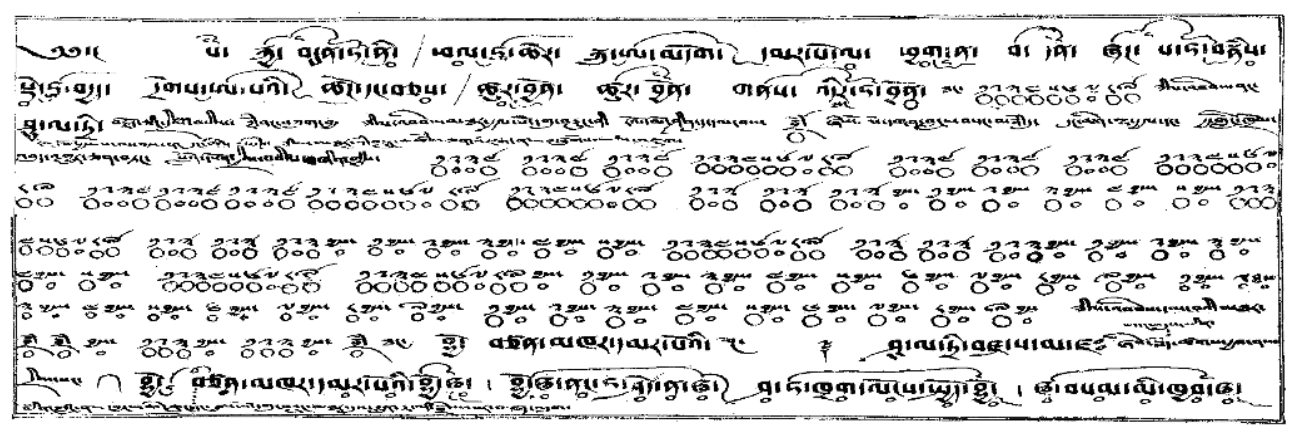

La séquence à 9 frappes (dgu-brdung) y est répétée neuf fois comme le met en évidence la transnotation proposée ci-après:

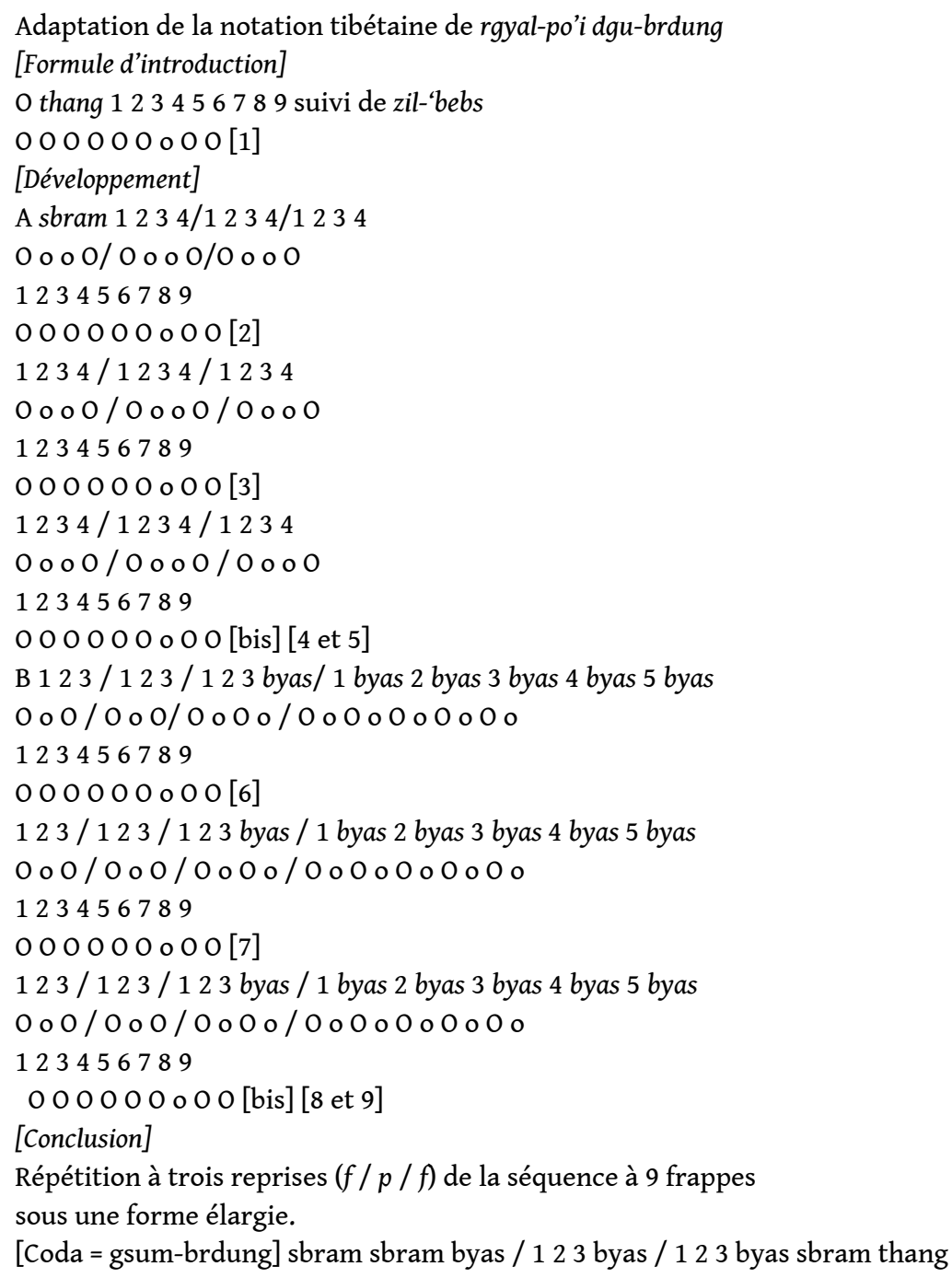

\section{Les interventions des instruments dans lesquels on souffle ('bud-pa)}

59 Ceux-ci - trompettes rkang-gling, longues trompes télescopiques dung-chen, hautbois rgyagling - sont toujours joués par paires d'instruments identiques; les dbyangs-yig indiquent 
bien où ils doivent intervenir, mais pour connaitre les modalités de leurs interventions, il faut consulter des recueils spécifiques, généralement manuscrits, comme ceux qui vont être succinctement décrits. Mais il faut surtout avoir recours à la tradition orale. On remarquera que chacun des répertoires considérés fait mention de sept pièces. Les experts consultés sur la raison de ce nombre régulier de compositions ont reconnu leur ignorance.

\section{Les sept formules pour le jeu des trompettes rkang-gling}

Que ces trompettes soient en os comme l'indique la terminologie (rkang-gling, «flûte en jambe») ou en métal, leurs interventions se limitent à de courtes formules composées d'un certain nombre de reprises de souffle, effectuées de la même façon quelle que soit la paire d'instruments utilisée et donc sans référence à une hauteur déterminée.

61 Le recueil manuscrit des notations, conservé au monastère de Shechen, se réfère à la tradition de Smin-grol-gling et distingue sept formules: trois variantes de «souffler à trois reprises» (gsum-'bud), trois variantes de « souffler à quatre reprises» (bzhi-bud), une formulation de "souffler à six reprises» (drug-bud) (cf. CD accompagnant Helffer 1994: pl. 14, d'après des enregistrements effectués à ma demande en 1981).

Les signes graphiques employés pour évoquer ces différentes formules sont peu nombreux et présentent un caractère à la fois prescriptif et descriptif: la prescription porte sur le nombre de reprises de souffles à effectuer, la description est suggérée par la forme des signes graphiques adoptés: un triangle allongé avec pointe vers le haut pour les sons à garder jusqu'à extinction, même figure tronquée pour un son qui doit être brusquement coupé (bcad-pa), prolongation de la pointe par une sorte de flamme pour indiquer que le son doit être violent et haché.

Un feuillet manuscrit de notations pour le jeu des mêmes trompettes, dites ici rkang-dung, provenant de Smin-grol-gling, m’a été généreusement donné par mon collègue et ami Alexander Macdonald qui l'avait acquis en 1961; ce document exceptionnel, que j'ai pu transmettre au monastère de Shechen, est intitulé « les façons de souffler les trompettes: voix rugissante des démons srin-po» (rkang-dung 'bud-tshig srin-po'i ngar-skad); il fournit une liste un peu différente des sept formules précédentes et a l'intérêt de préciser les conditions d'emploi de ces formules. Il distingue en effet: - Trois variantes de gsum-'bud, selon qu'elles interviennent pour l'invitation aux protecteurs de la religion, l'invitation aux divinités paisibles (zhi-ba'i spyan-'dren), et l'offrande aux divinités paisibles (zhi-ba’i mchod-pa). Elles sont associées au jeu des cymbales sil-snyan.- Deux variates de bzhi-bud, associées au jeu des cymbales sbug-chal / rol-mo, à utiliser au début et à la fin de l'invitation aux divinités terribles (drag-po'i spyan-'dren).- Une formulation de "souffler à cinq reprises» (lnga-'bud) pour la propitiation des protecteurs. - Une formulation de « souffler à six reprises» (drug-'bud) pour la sommation ('gugs-pa) des mêmes divinités. (cf. CD Sounds of Tibet, pl. 9).

Les signes graphiques adoptés s'inspirent nettement des notations neumatiques adoptées pour les chants dbyangs. 
Fig. 8: reproduction du feuillet de notations pour le jeu des rkang-gling selon la tradition de Shechen
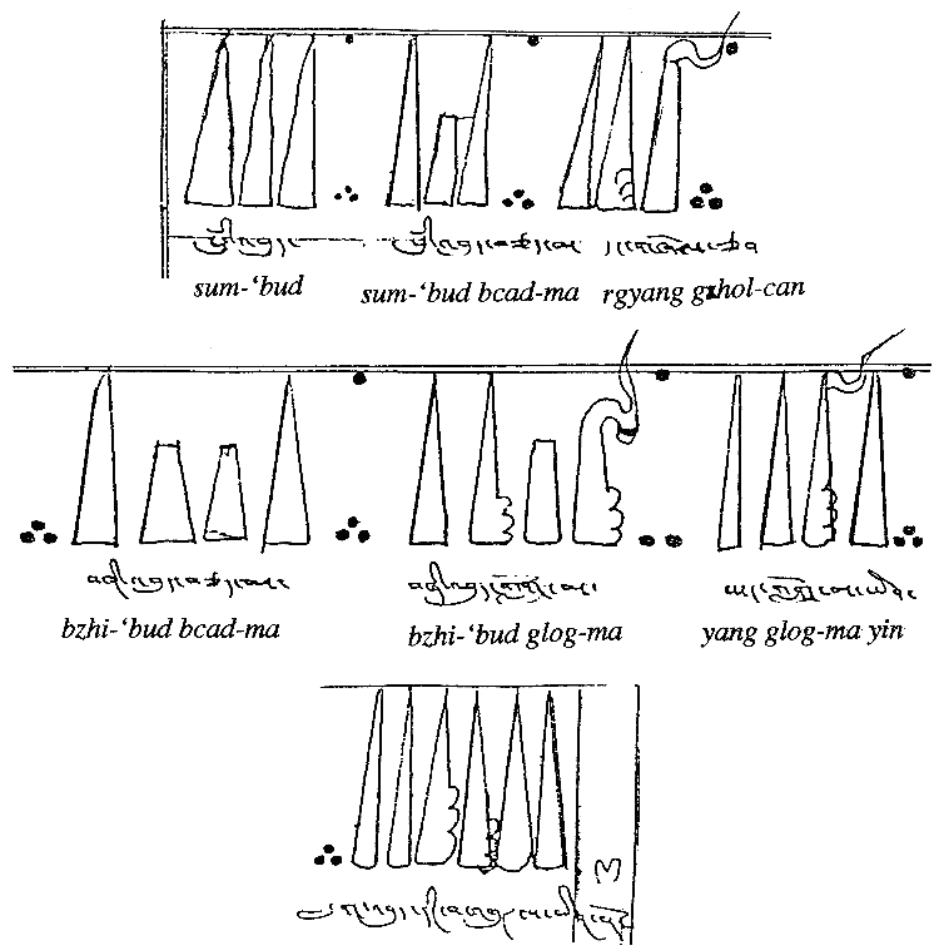

drug-'bud ring-ba'gugs-pa yin-no

Fig. 9: Reproduction du feuillet de notations pour rkang-dung du monastère de Smin-grol-gling

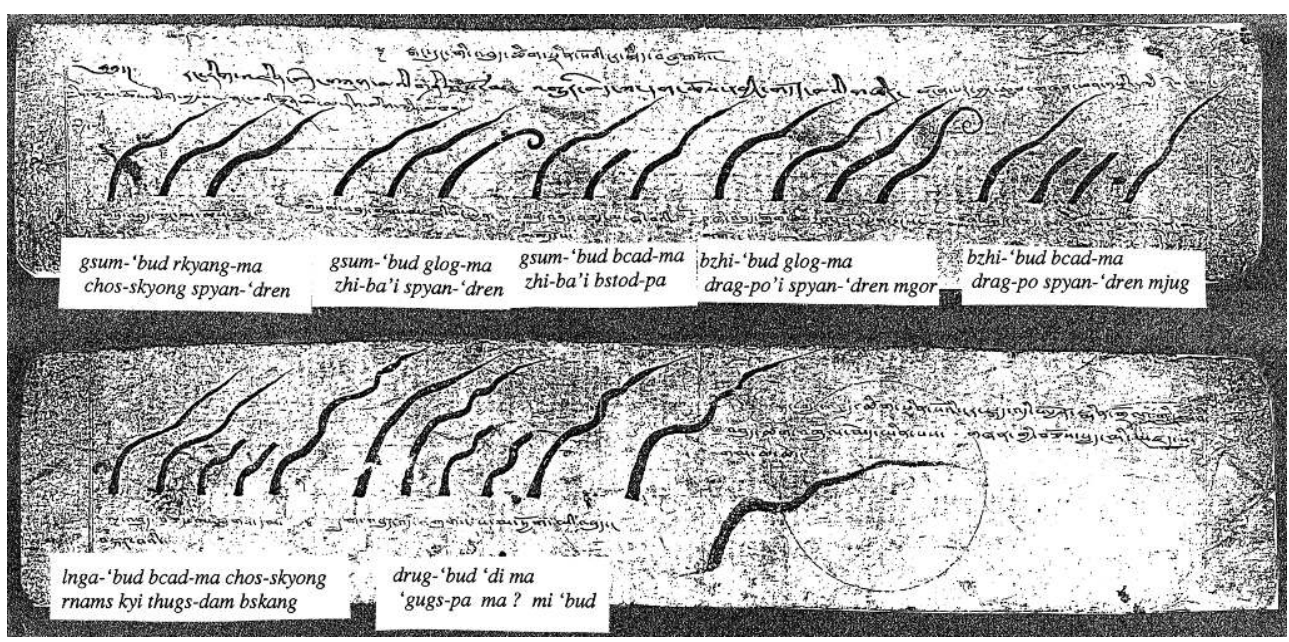

L'écoute des enregistrements confirme le fait que les prescriptions de ces recueils sont bien observées dans la pratique; il s'avère en particulier que, au cours de la propitiation ( bskang-ba) des divinités protectrices, la formule à cinq reprises de souffle avec coupure ( lnga-'bud bcad-ma) a bien été exécutée lorsqu'était mentionnée l'offrande du son du rkanggling. (cf. CD Sounds of Tibet pl. 6)

C'était le cas pour la propitiation de Mgon-po legs-ldan avec le quatrain qui suit:

Sha chen khrag dang dbang-po lnga

Par la chair humaine, le sang, les cinq organes des sens,

Tshil-chen shun-mar mkhris dang

par la graisse humaine, le beurre fondu et la bile, 
g.yang-gzhi lhab-lhub rkang-gling sgra

par une peau humaine flottant au vent, par le son de la trompe en os

dpal-mgon legs-ldan thugs-dam bskang

que soit loué le Glorieux protecteur.

66 Même énoncé de lnga-'bud bcad-ma lorsque un texte similaire exprimait les offrandes destinées à propitier la « Grand-mère gardienne des Mantras» (Ma-mo sngags-srung-ma); le son du rkang-gling étant cette fois associé à celui du tambour en crânes:

Mkhris-chen bdud-rtsi'i dri-chab dang

Par cette bile humaine au parfum d'ambroisie

Sha-khrag rus-pa'i zhal-zas dang

par cette nourriture de chair, sang et os

Rkang-gling thod-rnga'i rol-mo sogs

par la musique de la trompette en os et du tambour en crânes

Mthun-pa'i rdzas kyis thugs-dam bskang

par ces substances appropriées, qu'elle soit louée.

67 Il en allait de même lors de la propitiation de la «Grand-mère qui sévit dans les cimetières», pour laquelle l'accumulation des offrandes requises culmine avec le son du rkang-gling:

Dgra-bgegs srog-rtsa'i khrag dang dbang-po lnga

Tshil-gsur zhun-chen mar-nag sgron-me spar

Khrag zhag dri-chab sha rus don snying lnga

Rkang-gling rol-mor bcas-pa thugs-dam bskang

\section{Les sept pièces pour les longues trompes télescopiques dung-chen}

Ces trompes dont les tailles sont variables - la plupart des monastères en possèdent des « grandes» (dont la taille peut dépasser $3 \mathrm{~m}$ ) et des « petites», plus transportables (dont la taille avoisine 1,70m) - sont utilisées dans des contextes différents: solo pour accueillir un dignitaire (suites snyan-gsan) ou annoncer l'arrivée de danseurs pendant les ballets rituels 'cham et surtout participation à l'ensemble instrumental monastique, en liaison avec une composition pour cymbales et tambours. 
Fig. 10: Deux joueurs de trompes dung-chen dans la cour du monastère de Shechen

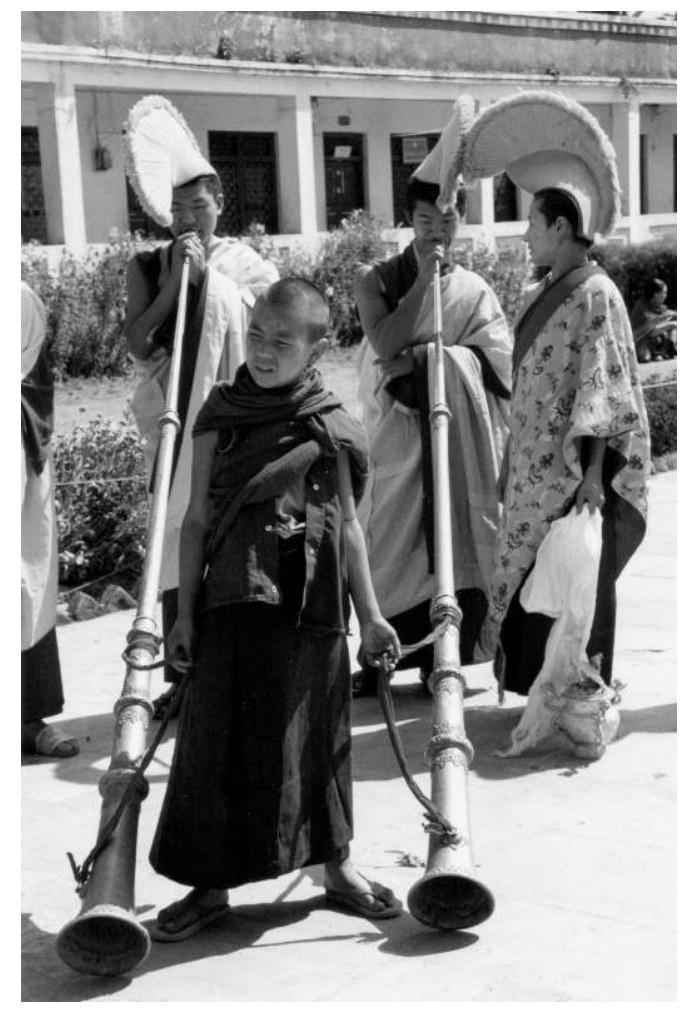

69 A Shechen, comme dans tous les monastères du bouddhisme tibétain, l'usage qui en est fait repose sur l'émission de trois hauteurs, désignées en tibétain par les termes 'dor pour le son grave, rgyang pour le son «étendu», à un octave du 'dor, ti/ni pour le son aigu, généralement à la quinte du rgyang.

70 Le répertoire, consigné dans un manuscrit peu soigné qui se compose de sept folios écrits recto/verso (S.T. 6), fournit une notation pour sept pièces de longueurs inégales. Dans le texte introductif qui précède les notations, il est précisé qu'il s'agit là d'une tradition conforme à celle de Smin-grol-gling (ce que confirme la consultation du recueil (dkar-chag ) où sont consignés les us et coutumes de Smin-grol-gling (S.T.4 /156).

71 Ces sept pièces sont désignées par des noms qui correspondent le plus souvent aux syllabes qui sous-tendent les signes de notation et qui servent de référence mnémotechnique aux musiciens:

- La première d'entre elles, intitulée Lha-rgyal-ma, s'appuie sur les syllabes bien connues, criées au passage des cols: Lha rgyal-lo ! Victoire aux dieux. Son exécution est préconisée dans le dbyangs-yig.

- La deuxième, intitulée "Le grand miséricordieux» Thugs-rje chen-po, réfère au bodhisattva Avalokiteshvara (tib. Spyan-ras-gzigs); les signes de notation sont soustendus par les syllabes Thugs-rje-chen répétées plusieurs fois, suivies de l'énoncé du célèbre mantra en six syllabes d'Avalokiteshvara OM MANI PADME [HUM], et complétées par le vœu que tous les êtres parviennent à la libération ('gro drug sems-can lam la sgrol / thar lam la drongs).

- La troisième, intitulée Rdo-rje sgra-dbyangs semble référer à une déesse de ce nom. Les syllabes qui sous-tendent les signes de notation correspondent à un texte difficile à établir en raison des nombreuses répétitions, mais qui renvoie explicitement à un des 
tantras les plus importants de l'école nyingmapa, à savoir le Sgyu-'phrul dra-ba ( = « le Filet d'illusion») Rdo-rje sgra-dbyangs lha-mo / kun-bzang rol-mo'i sprin-phung / sgyu-'phrul dra-ba'i dkyil-"khor lha-tshogs la spros-te phul dbyangs kyi rol-mo spros //

- La quatrième Dpal-mgon lcam-'dral renvoie à la forme du protecteur Mgon-po désigné par cet épithète et l'invite à venir pour accomplir les œuvres correspondant aux quatre catégories d'actions rituelles (las-bzhi): dpal-mgon lcam-dral 'dir byon / las-bzhi'i phrin-las thegs sgrub du gsol-bar mjod.

- La cinquième Rgyal-ba'i bstan-srung dam-can rgya-mtsho renvoie à l'océan des «protecteurs» de la doctrine bouddhique.

- La sixième Dam-can go-thog évoque la soumission des « liés par serment» (dam-can) qui ont fait le vœu de protéger la doctrine bouddhique, avec la succession dam-can bsrung-ma go-thog répétée plusieurs fois.

- La septième Bkra-shis 'khyil-ba «Enroulement de bon augure» est articulée sur les syllabes bkra-shis gad zhig zhing 'dir dpal 'bar / bkra shis gad zhig zhing 'dir 'khyil dont je ne comprends pas le sens.

Le nombre des signes de notation relatifs aux hauteurs à émettre se limite à huit, diversement combinés selon les pièces considérées qui commencent toutes par la succession 'dor-dor-rgyang et se terminent toutes par 'dor-rgyang.

Le développement des différentes pièces s'effectue à grand renfort de répétitions concernant le passage du son 'dor au son rgyang, avec de rares appels au son aigu $n i / t i$, toujours atteint en venant de rgyang exécuté ff et suivi d'une coupure. En fait, c'est le son 'dor qui marque les articulations des phrases.

On ne constate la présence d'aucune indication relative aux durées à respecter. Une description plus précise de la notation de lha-rgyal apportera néanmoins quelque lumière sur les enchaînements qui président à la composition de cette pièce emblématique.

Les indications manuscrites ajoutées aux courbes neumatiques, souvent difficiles à déchiffrer, portent sur deux points: d'une part le nom des hauteurs à émettre: le son grave 'dor ou le son aigu ni, d'autre part sur les syllabes d'un texte dont l'énoncé va accompagner l'émission du son rgyang, chaque syllabe correspondant à un signe neumatique distinct. Ce qui peut être traduit ainsi avec en italiques le nom des hauteurs et en majuscules la formule mnémotechnique à retenir par les musiciens:

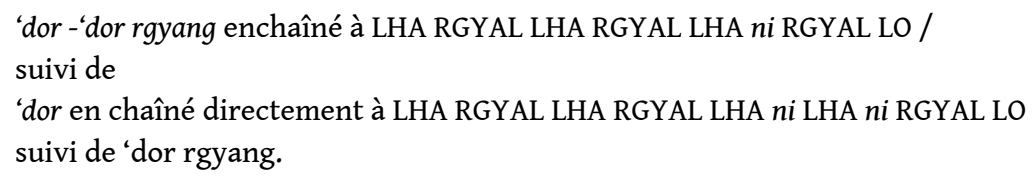

Les enregistrements de lha-rgyal réalisés à Shechen à plusieurs années d'intervalle avec des joueurs différents ont montré la stabilité de la forme générale de la pièce, mais une grande élasticité des durées: 10'12 en 1991 et 13'04 en 1997. L'exécution de lha-rgyal, la première pièce $\mathrm{du}$ répertoire, commune d'ailleurs à de nombreuses traditions monastiques (Helffer 1994: 53 tableau 8), qui figurait au début de la suite snyan-gsan enregistrée en 1997 n'a pu être conservée pour la publication du CD Sounds of Tibet en raison de sa qualité technique insuffisante. 
Fig. 11: Notation de Lha-rgyal selon la tradition du monastère de Shechen

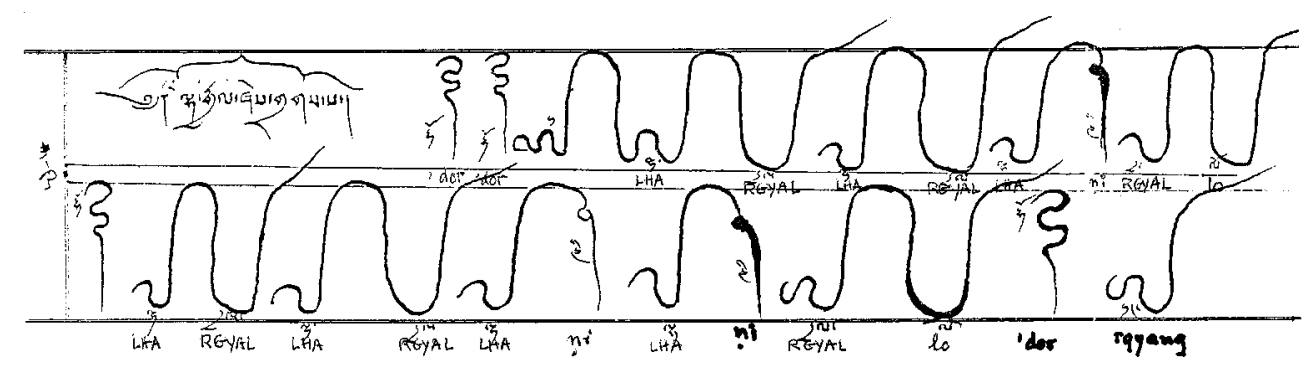

Que cette pièce présente un caractère emblématique me semble confirmé par le fait qu'il en existe une notation imprimée - provenant du monastère-référence de Smin-grol-gling - qui est reproduite ci-dessous, mais dont je ne suis pas en mesure de fournir une explication complète. Je remarque néanmoins que les enchaînements préférentiels de la fig. 11 y sont observés et que la prescription concernant l'emploi des sons 'dor, rgyang (indiqué ici par l'expression "voix naturelle» rang-skad) et ni ne s'écartent guère du modèle décrit ci-dessus.

Enfin, toujours concernant lha-rgyal selon la tradition de Smin-grol-gling, un assez long texte décrit les enchaînements dont il est composé et mériterait un examen attentif avec un joueur de ce monastère (S.T.5: 143).

79 C'est encore lha-rgyal qui avait été choisi par le maître de chant du monastère de Phyang au Ladakh pour illustrer le film de deux jeunes chercheurs hollandais Robert BoonzajerFlaes et Maarten Rens: 'dor low is better et l'on avait la surprise d'y entendre clairement l'énoncé des hauteurs à émettre, accompagné d'une gestuelle chironomique inspirée par le système de notation de ce monastère.

80 Je ne peux que souscrire aux remarques judicieuses d'Ivan Vandor quand il estime que l'architecture des pièces pour dung-chen est conçue par «juxtaposition de groupes symétriques»; il en propose même deux notations sur portée (Vandor 1976: 88-89), tout en reconnaissant que «La transcription de ces morceaux ne donne qu'une parmi les exécutions possibles, car le rapport entre les deux exécutants, tout en étant basé sur la même partie musicale, admet toutefois des différences de détails dans l'exécution. [...] On ne donne pas d'importance à ces différences, pourvu que l'allure générale du morceau soit respectée dans ses notes et leur durée, ainsi que dans ses pauses».

Fig. 12: Notation de Lha-rgyal selon la tradition du monastère de Smin-grol-gling

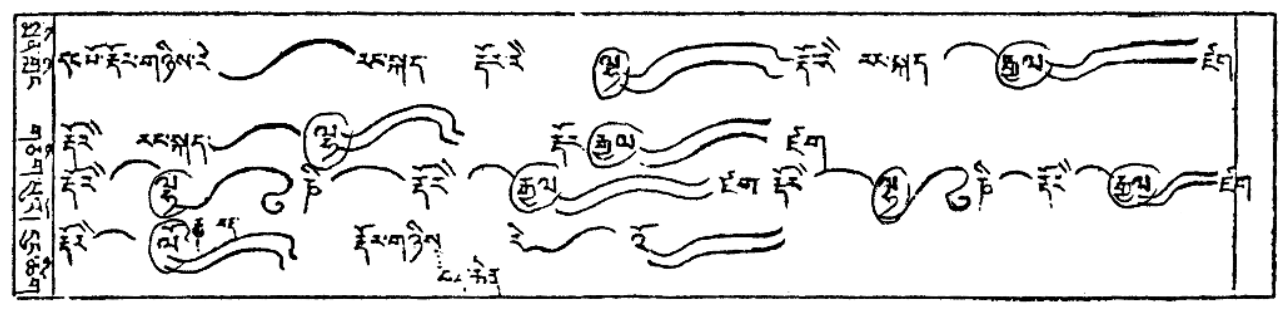

\section{Les sept pièces pour les hautbois rgya-gling}

81 Rappelons pour commencer quelques traits de la facture des hautbois rgya-gling: ils sont composés d'un tuyau en bois de perce cônique, pourvu de sept trous équidistants sur la 
face antérieure plus un trou sur la face postérieure et munis d'un pavillon plus ou moins évasé en métal. L'embouchure, à la base de laquelle est inséré un disque de métal, se compose d'un mince tuyau de métal dans lequel est insérée l'anche double (pi-pi). Ce dispositif favorise la technique de jeu qui fait appel à la respiration continue. Ce sont les deuxièmes phalanges des index, majeur et annulaire de la main proximale («main du haut» lag gong-ma) et index, majeur, annulaire et accessoirement auriculaire de la main distale ( « main du bas» = lag 'og-ma) qui sont utilisées pour obturer les trous de jeu. Seul le premier registre de l'instrument est utilisé et donc l'ambitus couvert par les joueurs de rgya-gling ne dépasse jamais la septième.

D'après le livret des études (sgrigs-gzhi) du monastère de Shechen qui m’a été montré par un moine, les pièces suivantes sont au programme: bla-ma mkhyen / mkha'-'gro gtso-mo (dont l'usage est préconisé en S.T.1: 418) / khrom-'bud 'gyur-ma (dont l'usage est préconisé en S.T.1: 339 et 361) / bung-ma (S.T.1: 346) / rdzogs rgan-ma 'am mdo-'bud / rdzogs gsar-ma / shri chen et shri chung //. La plupart de ces pièces figurent dans les enregistrements réalisés à ma demande en 1993, où sont enchainés les titres suivants:

Bla-ma mkhyen-no « hommage au lama» / 'Khrom-'bud 'gyur-ma / mkha'-'gro gtso-mo / bung-ma « l'abeille» / « don-grub» ( correspondant probablement à khrom-'bud ?) / shri chen et shri-chung (pour les prières du soir) / 'dra 'bud ring-thung sna-tshogs / Rgya-'bud, pièces de style chinois, jouées pour la fête de Padmasambhava lors de la célébration du $10^{\mathrm{e}}$ jour du mois (tshes-bcu), consacré à la mémoire des hauts faits de celui qu'on appelle aussi Guru Rinpoche.

Comme dans le cas des trompes dung-chen, selon les circonstances, ces pièces peuvent être jouées en solo - durant les processions ou au cours d'une suite snyan-gsan - ou en combinaison avec une composition pour cymbales et tambours comme on peut le constater à maintes reprises. Certaines d'entre elles sont jouées plus fréquemment, tel est le cas pour 'khrom-'bud 'gyur-can (CD Sounds of Tibet: pl. 4) ou mkha'-'gro gtso-mo (CD Sounds of Tibet pl. 12), pièce sur la composition de laquelle nous reviendrons ci-dessous (fig. 14).

En l'absence d'une notation propre à Shechen pour le jeu des hautbois rgya-gling, seule la simple écoute des documents sonores a permis de dégager certains traits de la composition des différentes pièces où abondent des répétitions multiples de séquences formées de courtes cellules mélodiques dont la structure semble découler de la facilité des doigtés (enchaînant comme on le verra des degrés conjoints).

Dans les rares cas où il a été possible de comparer plusieurs enregistrements d'une même pièce, il m'a toujours semblé qu'il y avait similarité du profil mélodique et donc conservation de la forme, quelles que soient les hauteurs absolues et l'élasticité des durées qui pouvaient être constatées. Mais la confirmation de ce point mériterait une étude approfondie que je n'ai pu entreprendre jusqu'à maintenant.

Je me limiterai donc à l'examen de deux exemples enregistrés en situation pendant le gtor-zlog en 1997 et figurant sur le CD Sounds of Tibet. Pour la présentation qui en est faite, je suis redevable à la compétence de Mikhail Malt, conseiller pédagogique à l'IRCAM, auquel je suis heureuse de dire ma reconnaissance pour l'inlassable intérêt qu'il a manifesté à l'égard de mes recherches; c'est grâce à lui qu'ont été mises en forme les notations des figures 13 et 14 obtenues par l'usage combiné de différents programmes informatiques (acousmographe, open music etc). Les dispositions adoptées ont pour but de mettre en évidence les quelques cellules mélodiques, arbitrairement identifiées au cours des écoutes répétées des documents sonores. 
Déjà, dans une publication antérieure (Helffer 1994: 78-79) j'avais tenté, à partir d'enregistrements faits en 1991, de montrer les variantes d'une courte séquence de hautbois correspondant à l'énoncé de la formule rythmique à trois frappes (gsum-brdung); la même démarche effectuée pour la même section rituelle exécutée en 1997 (CD Sounds of Tibet: plage 6) a permis de proposer la notation qui suit, dans laquelle les quatre énoncés retenus s'organisent autour de trois courtes cellules notées respectivement ré\#-mi-sol / mi-sol-la / si-sol-la, avec simplification progressive d'énoncé en énoncé; les traits verticaux qui découpent les lignes de notation ont été tracés par rapport à la figure rythmique énoncée, mais montrent bien la liberté qui préside à l'énoncé de la séquence de hautbois.

Si je me reporte maintenant à la plage 12 du CD Sounds of Tibet (durée: 3'54) qui correspond à la section finale du gtor-zlog (mais est également exécutée pour d'autres rituels), je constate que la composition, conformément aux prescriptions du manuel de notation, juxtapose non seulement une importante pièce pour les cymbales / tambours, mais aussi les hautbois et les trompes dung-chen. En raison de problèmes techniques, liés aux propriétés acoustiques des différents instruments, il n'a pas été possible d'obtenir une notation satisfaisante de l'ensemble ainsi constitué et je me limiterai ici à une tentative d'analyse de la composition jouée par les hautbois.

Fig. 13: Notation de plusieurs énoncés d'une séquence de hautbois correspondant à l'exécution de la formule rythmique gsum-brdung
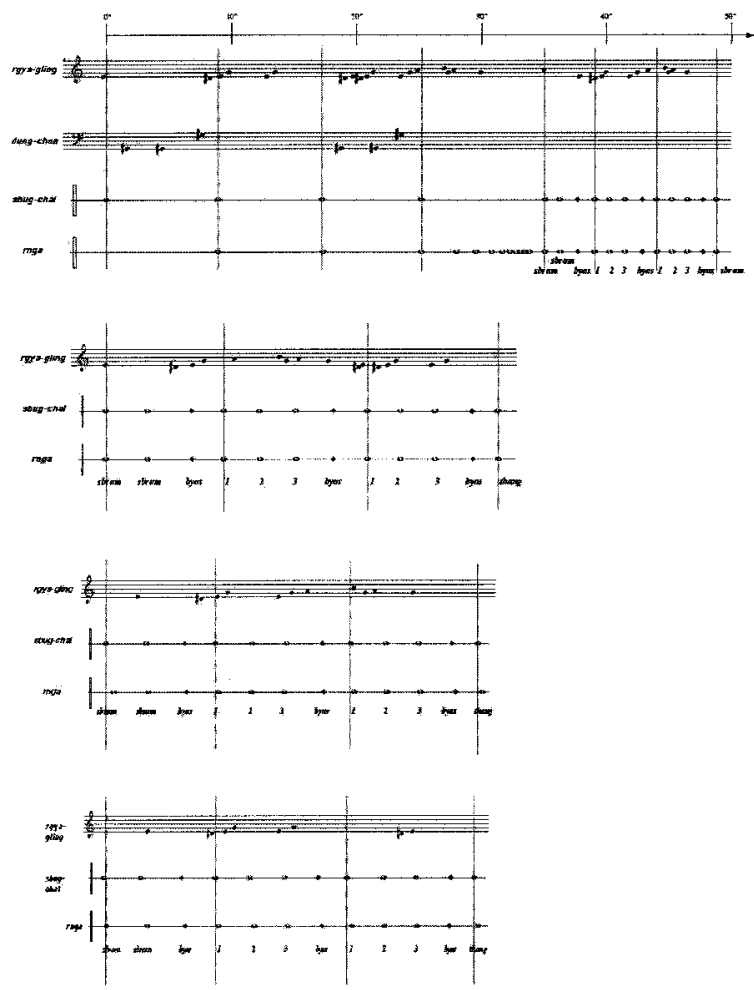

Cette pièce qui, selon les informateurs (et les indications concordantes du dbyangs-yig), s'intitule « la plus noble des dâkini(s) « (mkha'-'gro gtso-mo) repose (comme les séquences de la fig. 13) sur l'agencement de cellules qui introduisent par paliers successifs, en mouvement ascendant, les six hauteurs utilisées, notées respectivement <mi-fa\#- la-si do\#-ré\#>. La division en cellules adoptée, basée sur les répétitions observées, distingue: 
- Une cellule initiale dans le registre grave, basée sur les hauteurs mi-fa\#-la et dont certains éléments peuvent être répétés.

- Une cellule fa\#-la-si qui reprend les deux derniers degrés de la cellule initiale et introduit le degré suivant si.

- Une cellule do\#-la-si, introduisant le do\# en broderie des deux derniers degrés de la cellule précédente et éventuellement prolongée par si ou la-si.

- Une cellule ré\#-do\#-ré\# qui peut être considérée comme une broderie autour du do \# atteint dans la cellule précédente et constitue le point culminant de la pièce qui se développe ensuite avec un retour au registre grave en empruntant les cellules précédemment définies.

Dans la notation proposée ci-dessous, chacune des cellules a été entourée d'un cadre, sans qu'il soit tenu compte des durées.

Il s'avère que, dans un court manuel de la tradition de Smin-grol-gling (S.T.8), sont énumérées et décrites sept compositions pour rgya-gling dont les titres ne correspondent malheureusement pas au répertoire de Shechen, mais qui confirment les observations faites sur le terrain selon lesquelles la transmission du répertoire de rgya-gling se fait par la reproduction des doigtés utilisés par la main du bas et la main du haut (Helffer 1994: 80-83). Il est d'ailleurs frappant de constater à quel point, lorsque deux musiciens jouent ensemble du rgya-gling, le musicien le moins expérimenté a les yeux fixés sur les doigts de son partenaire!

La terminologie utilisée dans ce manuel n'a pu jusqu'à présent être complètement élucidée et les musiciens de Shechen interrogés à ce sujet ne la connaissaient pas.

Fig. 14: Notation de Mkha'-'gro gtso-mo

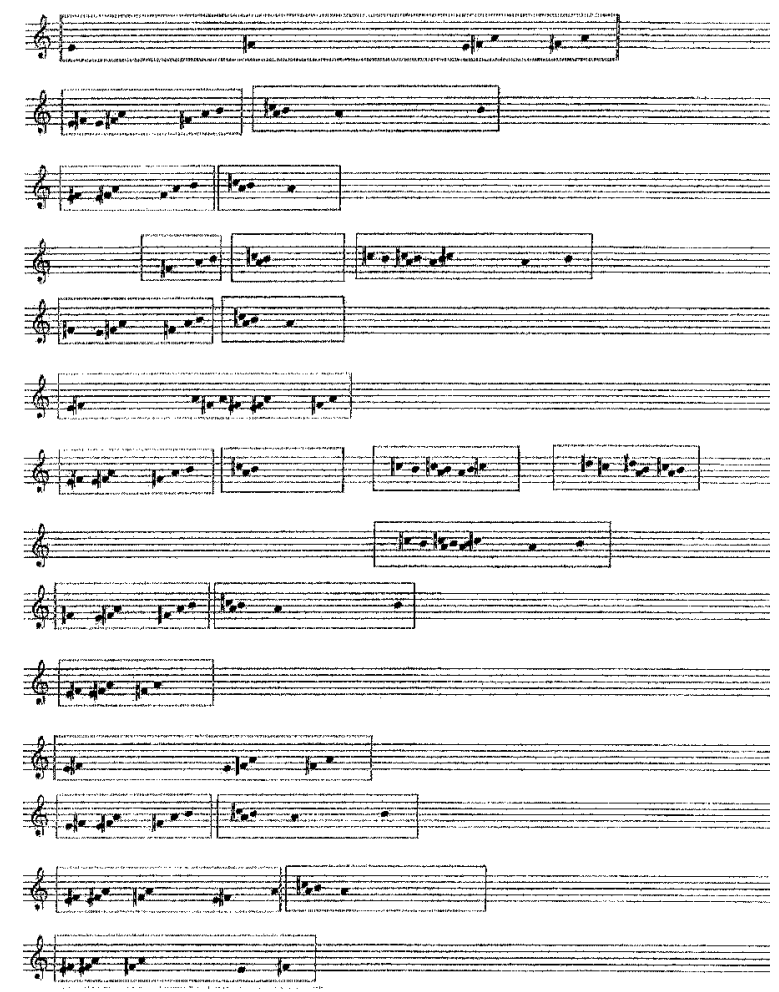

Pour des tentatives d'analyse des compositions pour hautbois rgya-gling selon d'autres traditions, on peut se reporter aux travaux de Crossley-Holland qui, à partir des 
enregistrements qu'il avait réalisés en 1961 lors de sonneries exécutées le matin et le soir (très probablement lors d'un snyan-gsan), propose une notation sur portée et une analyse détaillée des différentes cellules mélodiques dont la juxtaposition forme la trame des pièces en question (Crossley-Holland 1970).

\begin{tabular}{|l|l|}
\hline \multicolumn{2}{|l|}{ Répertoire de Smin-grol-gling d'après S.T.: 156 } \\
\hline $\begin{array}{l}\text { Les sept pièces pour } \\
\text { trompes dung-chen }\end{array}$ & $\begin{array}{l}\text { Les sept pièces pour } \\
\text { hautbois rgya-gling }\end{array}$ \\
\hline Lha-rgyl & bla-ma mkhyen-no \\
\hline Thugs-rje chen-po & khrung-'bebs \\
\hline Rdo-rje sgra-dbyangs & khra-skad \\
\hline Dpal-mgon lcam-dral & bung-ba \\
\hline Rgyal-ba'i bstan-srung & sva-ha \\
\hline Dam-can 'go-thog & btsun-mo chung-ba \\
\hline Bkra-shis 'khyil-ba & bkra-shis 'khyil-ba \\
\hline
\end{tabular}
malgré la pluie !), seulement six de ces pièces ont été exécutées, sans qu'il soit possible d'élucider les raisons du choix des musiciens: Lha-rgyal / bla-ma mkhyen-no / dam-can gothog / 'khrom-'bud / bkra-shis 'khyil-ba / bung-ma //

100 Toujours est-il que, quelles que soient les pièces choisies, on ne saurait demeurer indifférent à la magie qu'exercent les longs appels des trompes relayés par la stridence du jeu continu des hautboïstes. 


\section{Conclusion}

$101 \mathrm{Au}$ terme provisoire de ce regard sur les traditions musicales du monastère de Shechen, s'appuyant d'ailleurs, comme je l'ai signalé, sur celles du monastère de Smin-grol-gling, il s'avère qu'au cours du seul rituel pris en compte, de multiples manifestations sonores interviennent.

L'analyse des diverses composantes musicales qui contribuent à la magnificence et à l'efficacité des rituels et qui constituent «l'offrande musicale» (mchod-rol) présentée aux divinités permet, me semble-t-il, d'affirmer que les critères musicaux qui sont privilégiés sont essentiellement la forme générale et le timbre, au détriment des hauteurs et des durées.

Qu'il s'agisse de la composition des chants dbyangs ou du répertoire instrumental, il y a agencement de courtes cellules préexistantes plutôt que développement à proprement parler, et il paraît légitime de parler à ce propos d'un mode de composition par centonisation.

Pendant la performance, chaque instrumentiste ou chaque groupe d'instrumentistes se doit d'exécuter sa partie selon les modalités prévues par les manuels de notation et/ou par la tradition orale.

105 Lorsque plusieurs groupes d'instruments jouent simultanément, ils doivent normalement s'aligner sur le jeu des cymbales/tambours qui fournissent le cadre général; même si les départs des uns et des autres peuvent être indiqués de façon précise (geste du maître de chant ou formule particulière jouée par les cymbales), on n'observe pas une véritable coordination entre les différentes parties musicales, et on assiste plutôt à une juxtaposition des répertoires individuels qui doivent perpétuellement s'ajuster aux impératifs du rituel. On pourrait presque dire qu'il y a primauté de l'exécution musicale ponctuelle sur la musique elle-même, et ceci explique qu'il faille souvent rester à un niveau descriptif d'une performance donnée, sans possibilité réelle de généraliser les résultats obtenus.

Seule la multiplication des études ponctuelles dans un monastère donné, mais aussi dans plusieurs monastères d'une même tradition et, au-delà, dans les monastères des autres traditions et chez les bonpos, permettra de dégager si l'on peut parler d'un même fond commun de traditions musicales dans le monde tibétain.

\section{Bibliographie sélective des publications en langues occidentales}

Action poétique 157, 1999: Tibet. 
Asian Music X-2, 1979: Tibet issue.

CANZIO Riccardo O., 1978, Sakya Pandita's « Treatise on Music» and its relevance to present-day Tibetan Liturgy, Unpublished Ph.D thesis submitted to the School of Oriental and African Studies, University of London.

CANZIO Riccardo O., 1979, « On the way of Playing the Drums and Cymbals among the Sakyas». Tibetan Studies in honour of Hugh Richardson [Proceedings of the International Seminar on Tibetan Studies / Oxford 1979], Michael Aris \& Aung San Suu Kyi eds, Warminster, Aris and Phillips: 67-72

CANZIO Riccardo O., 1985, « Aspects of Ceremonial Behavior in Bon-po Monastic Life». Soundings in Tibetan Civilization [Proceedings of the 1982 Seminar of the IATS held at Columbia University], Barbara Nimri Aziz and Matthew Kapstein eds: 42-52.

CANZIO Riccardo O., 1986, « The Bonpo Tradition: Ritual Practices, Ceremonials, Protocol and Monastic Behaviour. An ethnomusicological description». Zlos-gar. Performing Traditions of Tibet, Jamyang Norbu ed., Dharamsala LTWA / 45-57.

CANZIO Riccardo O., 1988, «Etude d'une cérémonie de propitiation Bonpo -le Nag-zhig bskang-ba -; structure et exécution». Essais sur le Rituel I [Colloque du Centenaire de la section des Sciences religieuses de l'EPHE, Paris 1986], Anne-Marie Blondeau et Kristofer Schipper eds, Louvain-Paris, Peeters: 158-172.

CANZIO Riccardo O., 1991, Tibet: Traditions rituelles des Bonpos, 1 CD OCORA-Radio France C 580016 (enregistrements de l'auteur en mars 1981 et avril 1983); réédition augmentée de Les traditions rituelles des Bonpos tibétains, Festival de Lille / OCORA Radio France, LP 30cm/33t 558622 (1983). [Fournit l'enregistrement correspondant à Canzio 1988].

CROSSLEY-HOLLAND Peter, s.d. La musique du bouddhisme tibétain: Tibet I: The Nyingmapa and the Kagyupa Sects, Tibet II: The Sakyapa and Gelugpa Sects, Tibet III: The Kagyupa and the Gelugpa Sects, Barenreiter Musicaphon, UNESCO Collection: A Musical Anthology of the Orient, BM 30 L 2009-2011, 3 disques LP 30cm/33t.

CROSSLEY-HOLLAND Peter, 1981, « rGya gling hymns of the Karma-Kagyu. The rhytmitonal Architecture of some Instrumental Airs». Selected Reports in Ethnomusicology, vol. I/3: 79-114. [cf. A Musical Anthology of the Orient, UNESCO Collection TIBET I, Bärenreiter Musicaphon BM 30L2009: pl.12 et 13, enregistrées en 1961].

DEMIEVILLE Paul, 1976, « Notes on Buddhist Hymnology in the Far East», Buddhist Studies in honour of Walpola Rahula. London: Gordon Fraser: 44-61.

ELLINGSON Ter, 1979a, The Mandala of Sound: Concepts and Sound Structures in Tibetan Ritual Music. Ph.D in Philosophy of the University of Wisconsin-Madison. Fac simile printed in 1984 by University Microfilm International, Ann Arbor, Michigan.

ELLINGSON Ter, 1979b, « 'don rta dbyangs gsum.Tibetan chant and melodic categories». Asian Music $\mathrm{X} / 2: 112-158$.

ELLINGSON Ter, 1979c, « The mathematics of Tibetan Rol-mo». Ethnomusicology XXIII/2: 225-243.

ELLINGSON Ter, 1980, « Ancient Indian Drum Syllables and Bu-ston's Sham-pa-ta Ritual». Ethnomusicology XXIV/3: 431-452.

ELLINGSON Ter, 1986, « Buddhist Musical Notations». The Oral and Literate in Music, Tokumaru Yoshihiko and Yamaguti Osamu eds. Tokyo: Academia Music Ltd: 302-342.

ELLINGSON Ter, 1992, Articles « Transcription» et « Notation». Ethnomusicology, an Introduction [The New GROVE Handbooks], Helen Myers ed.. London: The Macmillan Press: 112-152 et 153-164. 
FERRETTI Don Paolo, 1986, Esthétique grégorienne ou traité des formes musicales du Chant grégorien. Paris: Desclée.

HELFFER Mireille, 1976, « Traditions musicales des Sa-skya-pa relatives au culte de Mgon-po». Journal Asiatique CCLXIV: 357-404

HELFFER Mireille, 1977, LADAKH. Musique de monastère et de village. 1 disque LP 30cm/33t, Chant du Monde LDX 274662, coll. CNRS/Musée de l'Homme: Traditions musicales des cinq continents. Réédition augmentée, même collection, 1 CD CM 251 (1989).

HELFFER Mireille, 1980, « The musical notation of the hymn Rtsa-brgyud-ma in the Dge-lugs-pa tradition». Tibetan Studies in honour of Hugh Richardson [Proceedings of the International Seminar on Tibetan Studies /Oxford 1979], Michael Aris \& Aung San Suu Kyi eds, Warminster Aris and Phillips: 120-131.

HELFFER Mireille, 1987, « Preliminary remarks concerning the use of musical notations in Tibet». Zlos-gar, Dharamsala: Jamyang Norbu ed., LTWA: 69-90.

HELFFER Mireille, 1990, « Recherches récentes concernant l'emploi des notations musicales dans la tradition tibétaine». Tibet, Civilisation et Société. Colloque organisé par la Fondation SingerPolignac (Paris 1987), Fernand Meyer éd.: 59-84.

HELFFER Mireille, 1993, « An Overview of Western Work on Ritual Music of Tibetan Buddhism». European Studies in Ethnomusicology: Historical Developments and Recent Trends [Intercultural Studies 4], M.P. Baumann, A. Simon and U. Wegner eds. Wilhelmshaven: Florian Noetzel Verlag: 87-101.

HELFFER Mireille, 1994, Mchod rol. Les instruments de la musique tibétaine. Paris: CNRS éditions et Editions de la Maison des Sciences de l'Homme (avec CD encarté).

HELFFER Mireille, 1995, «Quand le terrain est un monastère bouddhique tibétain», Cahiers de musiques traditionnelles 8: Terrains: 69-84.

HELFFER Mireille, 1998, « Du son au chant vocalisé: la terminologie tibétaine à travers les âges (VIII $-\mathrm{XX}^{\mathrm{e}}$ siècle)», Cahiers de musiques traditionnelles 11: Paroles de musiciens: 141-162.

HELFFER Mireille, 2004, « Traditions musicales dans un monastère du bouddhisme tibétain», L’Homme № 171-172: Musique et anthropologie.

HELFFER Mireille et Jean SCHWARZ, 1981, « De la notation tibétaine au sonagramme», Ethnomusicologie et représentations de la musique, hors série du numéro $42 \mathrm{du}$ Courrier du CNRS $\mathrm{N}^{\circ} 42$ (1981): 12-13.

HELFFER Mireille et Matthieu RICARD, 1997, Sounds of Tibet / Musique sacrée du monastère de Shéchen. 1 CD conçu et élaboré par M. Helffer et Matthieu Ricard. Delhi et Kathmandu: Shechen Publ. 001.

HUC Regis-Evariste, 1987, Souvenirs d'un voyage dans la Tartarie et le Thibet, 2 tomes. Paris: Peuples du Monde et Astrolabe (coll. Domaine Tibétain), réimpression de l'édition de Pékin 1924.

KAUFMANN Walter, 1982, Tibetan Buddhist Chant ${ }^{3}$. Bloomington-London: Indiana University Press. MESCHONNIC Henri, 1980, Critique du rythme; anthropologie historique du langage. Paris: Verdier.

ROUGET Gilbert, 1990, «La répétition comme universel du langage musical. A propos d'un chant initiatique béninois», in La Musica come Linguaggio Universale: Genesis e storia di un'idea, a cura di Rafaele Pozzi. Firenze: Olschki: 189-201.

SCHEIDEGGER Daniel A., 1976, Tibetan Ritual Music. A General Survey with Special Reference to the Mindroling Tradition. Rikon: Tibet-Institut [Opuscula Tibetana Fasc. 19]. 
TOURNADRE, Nicolas, 1986, « La métrique», Tibet / Action poétique 157 (1999): 18-23

TSUKAMOTO, Atsuko, 1982, « The Music of Tibetan Buddhism in Ladakh: the Musical Structure of Tibetan Buddhist Chant in the Ritual bskang-gso of the Dge-lugs-pa Sect». Yearbook for Traditional Music vol. XV: 126-136. [Enregistrements de référence faits par l'auteur en 1980 au monastère dge-lugs-pa de Spituk au Ladakh: 2 disques LP 30cm/33t Dkyil 'khor chos skyong gi bskang-gsol, Nippon G 2 Columbia 7158-59].

VANDOR Ivan, 1976, Bouddhisme tibétain. Paris: Buchet-Chastel, coll. Les Traditions musicales, vol. VII.

\section{Sources tibétaines (S.T.) citées}

S.T.1 Dpal rgyal ba Zhe chen pa'i lugs kyi dbyangs yig skor. A Collection of Musical Notations for the Rituals Performed at the Nyingma Monastery of Shechen Tennyi Dargyeling, New-Delhi, Shechen Publications, n.d., $642 \mathrm{p}$.

S.T. 2 Zhe chen lugs srol cho ga'i lag len skor. A set of unique manuals for the rituals in use at Shechen Tennyi Dargyeling monasteries, in accordance to the Mindroling tradition of the Nyingma School of Tibetan Buddhism, New Delhi, Shechen Publications, 2000, 2 vol. 192 p. + 190 p. [préface par Matthieu Ricard]

S.T. 3 Rol mo’i bstan bcos, [Traité de la musique de Sa skya Pandita et commentaire], Beijing, Mi rigs dpe skrun khang, 1985

S.T. 40 rgyan Smin grol gling gi dkar chag [Us et coutumes du monastère de Smin grol gling], Krung go'i Bod kyi zhes rig dpe skrun khang gyis bskrun, 1992.

S.T. 5 Gsang rnying rgyan dang rol mo'i bstan bcos [Compilation de textes relatifs à la musique et aux objets rituels], Bod ljongs Bod yig dpe rnying dpe skrun khang gis bskrun [Collection Gangs can rig mdzod № 30], 1996.

S.T. 6 Ms inédit: Dung tshig gi yi ge « Mchod sprin rnam par bkod pa'i rol mo», 7 fol. [Notation pour le jeu des trompes dung-chen, dite « Musique qui se répand comme un nuage d'offrandes»].

S.T. 7 Ms inédit: rkang-dung gi 'bud-tshig «srin-po'i ngar-skad», 1 fol. recto verso [notation pour souffler dans les rkang-dung dite « rugissement des démons srin-po»]

S.T. 8 Rgya-gling rtsa-tshig rab-'byams zhing kun-khyab-pa'i sgra-snyan zhes bya-ba bzhugs-so (titre abrégé: gling-tshig), 6 fol.; exposition of the method of playing the rgya-gling in Tibetan Buddhist ritual performance by Gter-sras Rinchen rnam-rgyal of Smin-grol-gling. Reproduced from a manuscript of Smin-grol-gling. Editions du monastère de Dpal-yul (en Inde), 1985.

\section{Enregistrements de référence déposés au Laboratoire d'ethnomusicologie du Musée de l'Homme (des copies en ont été fournies au monastère népalais de Shechen)}

CD 999.009.001: 1 à 9

Manuel des notations concernant le culte rendu aux chos-skyong (S.T.1: 267-425), enregistré par le dbu-mdzad Nor-bzang s'accompagnant aux cymbales. Bodnath, 1995.

CD 003.001. 01: 1 à 5

Cahiers d'ethnomusicologie, $17 \mid 2011$ 
Manuel des notations concernant le culte rendu à Gshin-rje-gshed (S.T 1: 509-630), enregistré par le dbu-mdzad Nor-bzang s'accompagnant aux cymbales, Bodnath, 1995

CD 002.018.001: 1 à 4

Rituel du gtor-zlog: session de l'après-midi, enregistré au Shechen népalais le $4^{\text {ème }}$ jour du rituel, 1995

CD $998.002 / 1$ à 7

Copie CD des enregistrements effectués sur DAT sous ma direction en 1997 et dont une sélection figure dans le CD Sounds of Tibet.

Rituel du gtor-zlog: session de l'après-midi du $5^{\mathrm{e}}$ jour

- CD 2: bskang-ba des protecteurs $466^{\prime} 03$

- CD 3: zor-'phrin 73’28

- CD 4: fin du zor-'phrin 26'

- CD 5: Cérémonies conclusives de fin de journée

S'y ajoutent:

- CD 6: Extraits du grand rituel du Tshogs-chen ‘dus-pa 50’32

- CD 7: Suite instrumentale snyan-gsan 58'14

- lha-rgyal (trompes dung-chen) 13'41

- bla-ma mkhyen-no (hautbois rgya-gling) 7'59

- dam-can go-thog (trompes dung-chen) 5'12

- khrom-'bud (hautbois rgya-gling) 12'11

- bkra-shis 'khyil-ba (trompes dung-chen) 8’34

- bung-ma (hautbois rgya-gling) 10'36

Répertoire des pièces pour hautbois rgya-gling dans la tradition de Shechen

Enregistré hors-situation à ma demande le 25/2/1993

(cassette originale 1993-8, copiée sur CD en 2002). Durée totale: 58'33

- bla-ma mkhyen-no 7'53

- khrom-'bud 'gyur-ma 9'34

- mkha'-'gro gtso-mo 9'05

- bung-ma $10^{\prime} 47$

- mdo 'bud ou rdzogs rgan-ma 9'37

- prière aux protecteurs version longue shri-chen suivie de version courte shri-chung 2'

- pour les offrandes 4'43

- pièce de style chinois (rgya-'bud) 3'25

CD accompagnant Helffer 1994

Pl. 8 3'15

Pl. 9 1'47 
Pl. 14: les formules pour le jeu des trompettes rkang-gling 2'30

Pl. 15: Extrait du rituel de Vajrasattva 2'09

\section{NOTES}

1. Des extraits des enregistrements réalisés figurent dans le CD accompagnant Helffer 1994 et des copies CD des plus importants d'entre eux ont été déposées au Laboratoire d'ethnomusicologie du Musée de l'Homme. On en trouvera les références à la suite de la bibliographie. D'un intérêt tout particulier est l'enregistrement intégral des deux manuels de notations utilisés pour le gtor-zlog, réalisé auprès du maître de chant (dbu-mdzad) Nor-bzang s'accompagnant aux cymbales en 1995: le manuel pour les chos-skyong (ci-dessus S.T.1: 267-425) / CD Musée de l'Homme 999.009: 1 à 9) et le manuel pour Gshin-rje-gshed (ci-dessus S.T.1: 509-630) / CD Musée de l'Homme 003.001: 1 à 5). S'y ajoutent un enregistrement des différentes formules pour le jeu des trompettes rkang-gling ( $c f . C D$ inséré dans Helffer 1994: pl.14) et un enregistrement du répertoire des pièces pour hautbois rgya-gling (cassette originale 1993-8). C'est finalement à l'instigation de Rabjam Rinpoche que Matthieu Ricard et moi-même avons préparé le CD Sounds of Tibet (1997), qui regroupe une sélection des enregistrements recueillis en 1997.

2. Format des publications faites en Inde: $36 \mathrm{~cm} 27,5 \mathrm{~cm}$.

3. J'ai indiqué des limites de cet ouvrage dans deux comptes-rendus: Fontes Artis Musicae 1977/4:299, et The World of Music vol. XVIII/3 (1976): 58-60

\section{RÉSUMÉS}

On trouvera ici une tentative pour élucider les formes qu'empruntent les diverses expressions musicales qui animent les liturgies du bouddhisme tibétain, selon la tradition du monastère nyingmapa de Shechen. Cette contribution, volontairement limitée à la tradition d'un unique monastère, s'appuie aussi bien sur la consultation des manuels de notations élaborés au monastère-mère (sans doute au cours du XIX ${ }^{\mathrm{e}}$ siècle) et publiés récemment en Inde que sur les nombreux enregistrements réalisés par l'auteur depuis 1989. Pour permettre une discussion des résultats obtenus, la plupart des exemples analysés figurent dans l'enregistrement du CD commercial Sounds of Tibet, publié sous l'égide du monastère.

Il s'agit d'un premier pas qui devrait permettre d'envisager dans le futur une comparaison avec les traditions d'autres monastères de la tradition nyingmapa, voire celles d'autres écoles religieuses $\mathrm{du}$ bouddhisme tibétain ou des bonpos pour aboutir éventuellement à une généralisation des principes mis en évidence qui reposent tous, me semble-t-il, sur la juxtaposition de petits éléments diversement agencés, autrement dit qui illustrent une forme de centonisation. 


\section{AUTEUR}

\section{MIREILLE HELFFER}

Mireille Helffer, directeur de recherche honoraire au CNRS, a contribué à la mise en place de l'enseignement de l'ethnomusicologie à l'Université de Paris X-Nanterre, où elle a enseigné jusqu'en 1992. Spécialiste des musiques himalayennes, elle a effectué de nombreuses missions au Népal, au Ladakh et parmi les communautés tibétaines de l'exil. Ses publications ont touché à des domaines aussi variés que les castes de musiciens au Népal, le chant épique tibétain, le répertoire liturgique des monastères du bouddhisme tibétain, les notations musicales tibétaines ou les instruments de la musique tibétaine. Elle se consacre aujourd'hui à l'étude des traditions musicales du monastère de Zhe-chen au Népal. 